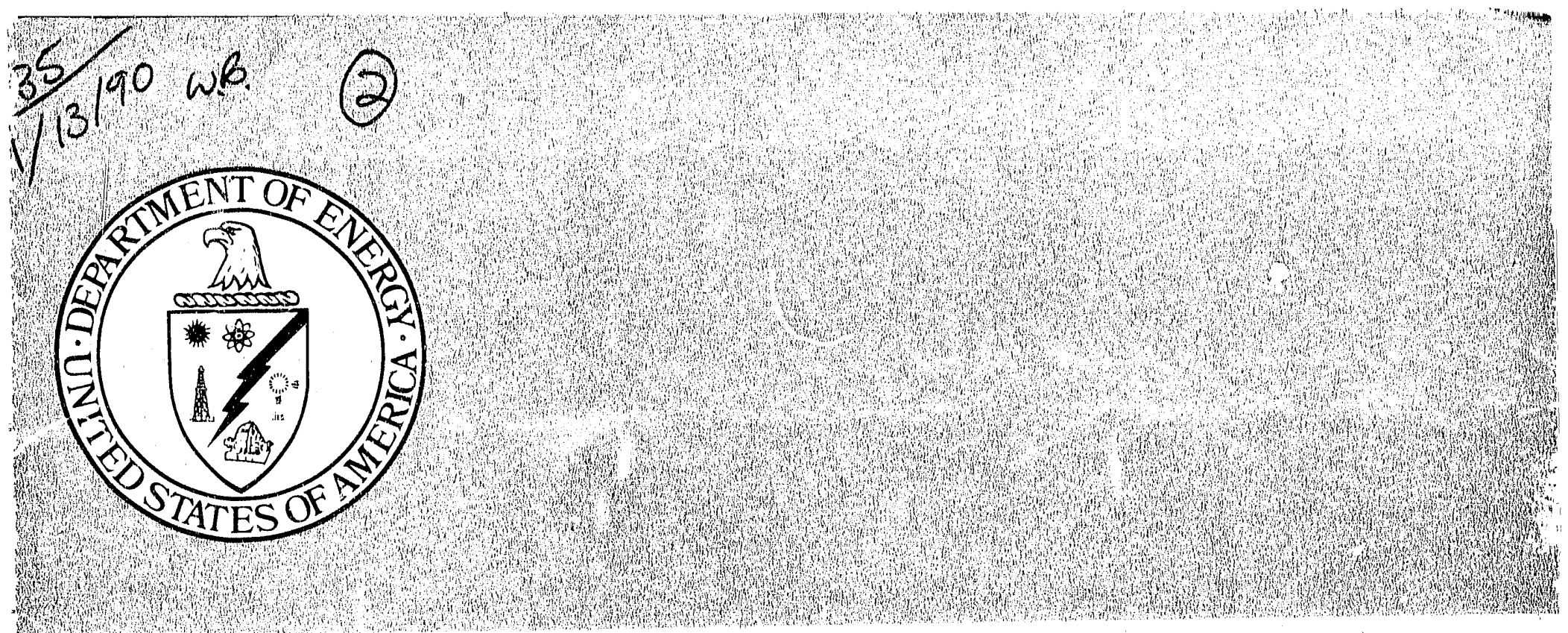

DOE/PETC/TR-90/9

(DE91000941)

A REVIEW OF INTERACTION MECHANISMS IN FLUID-SOLID FLOWS
By
G. Johnson
M. Massoudi
K. R. Rajagopal

September 1990

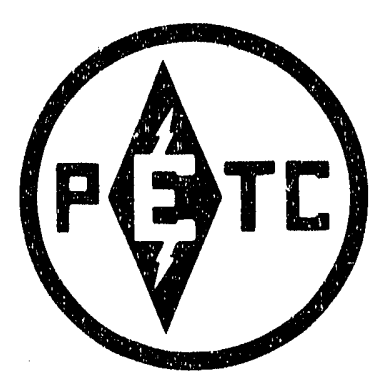

Pittsburgh Energy Technology Center

Pittsburgh, Pennsylvania 


\section{DISCLAIMER}

This report was prepared as an account of work sponsored by an agency of the United States Government. Neither the United States Government nor any agency thereof, nor any of their employees, makes any warranty, express or implied, or assumes any legal liability or responsibility for the accuracy, completeness, or usefulness of any information, apparatus, product, or process disclosed, or represents that its use would not infringe privately owned rights. Reference herein to any specific commercial product, process, or service by trade name, trademark, manufacturer, or otherwise does not necessarily constitute or imply its endorsement, recommendation, or favoring by the United States Government or any agency thereof. The views and opinions of authors ex. pressed herein do not necessarily state or reflect those of the United States Goverriment or any agency thereof.

This report has been reproduced directly from the best available copy.

Available to DOE and DOE contractors from the Office of Scientific and Technical Information, P.O. Box 62, Oak Ridge, TN 37831; prices available from (615).576-8401, FTS 626-8401.

Available to the public from the National Technical Information Service, U.S. Department of Commerce, 5285 Port Royal Rd., Springfield, VA 22161.

Price: Printed Copy A04

Microfiche A01 


\title{
A Review of Interaction Mechanisms in Fluid-Solid Flows
}

\author{
G. Johnson ${ }^{1} \quad$ M. Massoùdi K.R. Rajagopal ${ }^{2}$ \\ U.S. Department of Energy \\ Pittsburgh Energy Technology Center \\ P.O. Box 10940 \\ Pittsburgh, PA 15236-0940
}

\footnotetext{
${ }^{1}$ Department of Mechanical Engineering, University of Pittsburgh. Supported by an appointment to the U.S. Department of Energy Fossil Energy Laboratory Graduate Participation Program administered by Oak Ridge Associated Universities.

${ }^{2}$ Department of Mechanical Engineering, University of Pittsburgh, Pittsburgh, PA 15261
} 


\begin{abstract}
Multiphase flows have become the subject of considerable attention because of their importance in many industrial applications, such as fluidized beds, pneumatic transport of solids, coal combustion, etc. Fundamental research into the nature of pneumatic transport has made significant progress in identifying key parameters controlling the characteristics of these processes. "During the last few decades, many studies concerning the flow of fluid-solid mixtures have been published. Most of these studies rely almost entirely on empirical correlations, which generally compare poorly to experimental observations in all but the specific situations for which they are developed. While these approaches may be quite useful in predicting the global character of the flow system, they do not acount for local variations of solid concentration, velocity, and pressure or for velocity differences between the phases.

The two foremost approaches used in modeling multicomponent systems are averaging and mixture theory. In the first method, point-wise equations of motion are modified to account for the presence of the other components and the interaction between components. These equations are then averaged over time or some suitable volume, which is large compared with some characteristic dimension but small compared to the dimensions of the whole system. The second approach used in the mathematical modeling of multicomponent systems is mixture theory, which is a means for studying the interaction between several constituents by generalizing the equations and principles of the mechanics of a single continuum. In both approaches, constitutive relations are required to represent the interactive forces and the stress tensors for each constituent.

In this work, we review the existing constitutive relations for the interactive forces. The emphasis of this study is on a mixture composed of spherical particles of uniform size and a linearly viscous fluid. Section 1 introduces our approach and the importance of this study. In Section 2, the dynamics of a single particle as studied in classical hydrodynamics and fluid dynarnics is presented. This has been a subject of study for more than 200 years. In Section 3, we review the literature for the constitutive relations as given in multiphase studies, i.e., generalization of single particle and as given in literature concerning the continuum theories of mixtures or multicomponent systems. In Section 4, a comparison between these representations and the earlier approach, i.e., forces acting on a single particle will be made. The importance of flow regimes, particle concentration, particle size and shape, rotation of the particle, effect of solid walls, etc. are discussed. In Appendix A, a brief review of the "principle of material frame indifference" as used in modern continuum mechanics is provided. Examples of frame-indifferent and frame-dependent forms of the relative acceleration (related to the virtual mass force) are also given. In Appendix B, an "order-of-magnitude" analysis and comparison for various interactive forces are provided.
\end{abstract}




\section{LIST OF SYMBOLS}

\begin{tabular}{|c|c|}
\hline$a$ & sphere radius \\
\hline $\mathbf{a}_{u m}$ & relative acceleration \\
\hline Ac & acceleration number \\
\hline$C_{D}$ & drag coefficient \\
\hline$C_{H}$ & Basset coefficient \\
\hline$C_{u m}$ & virtual mass coefficient \\
\hline $\mathbf{D}_{f}$ & rate of deformation tensor of the fluid \\
\hline$f_{B}$ & buoyancy force, bold face denotes vector \\
\hline$f_{B 0}$ & single particle buoyancy force, bold face denotes vector \\
\hline$f_{\text {df }}$ & diffusion force, bold face denotes vector \\
\hline$f_{D}$ & drag force, bold face denotes vector \\
\hline$f_{D .}$ & single particle drag force, bold face denotes vector \\
\hline$f_{F}$ & Faxen force, bold face denotes vector \\
\hline$f_{H}$ & Basset force, bold face denotes vector \\
\hline$f_{I}$ & total interaction force, bold face denotes vector \\
\hline$f_{p}$ & pressure force, bold face denotes vector \\
\hline$f_{s}$ & total force on a particle, bold face denotes vector \\
\hline Jvm & virtual mass force on a particle, bold tace denotes vector \\
\hline$g$ & acceleration of gravity \\
\hline$i$ & $\sqrt{-1}$ \\
\hline $\mathbf{I}$ & indentity tensor \\
\hline$I_{\mathrm{D}}$ & first principle invariant of $\mathrm{D}$ \\
\hline$I I_{\mathrm{D}}$ & second principle invariant of $\mathbf{D}$ \\
\hline$I I I_{\mathbf{D}}$ & third principle invariant of $D$ \\
\hline$k$ & velocity gradient in simple shear \\
\hline$K$ & magnitude of the curl of the fluid velocity field \\
\hline $\mathbf{K}$ & curl of the fluid velocity field \\
\hline$m_{f}$ & mass of fluid displaced by solid particle \\
\hline$m_{\mathrm{s}}$ & mass of solid particle \\
\hline$m_{v m}$ & added (or virtual) mass of solid particle \\
\hline$p_{f}$ & fluid pressure \\
\hline Re & Reynolds number \\
\hline$t$ & time \\
\hline$t_{1}$ & dummy variable of integration \\
\hline $\mathrm{T}_{f}$ & fluid stress tensor \\
\hline$u$ & solid (distributed) phase velocity, boldface denotes vector \\
\hline$u_{t}$ & local acceleration of a particle \\
\hline$v$ & fluid (continuous) phase velocity, boldface denotes vector \\
\hline$v_{c}^{*}$ & reference velocity \\
\hline$v_{\tau}$ & relative velocity between phases, boldface denotes vector \\
\hline
\end{tabular}


$V_{A} \quad$ volume of fluid above the particle

$V_{\text {s }} \quad$ volume of particle

W spin tensor

$\mathbf{x}$ current configuration

$\mathbf{x}^{*} \quad$ current configuration, new frame

$\mathrm{X}$ reference configuration

$\mathbf{Y}$ position of sphere center

e void fraction

$\theta$ temperature

$\lambda$ parameter in relative acceleration expression, function of volume fraction

$\Lambda$ weighting function for virtual mass force

$\mu_{f} \quad$ fluid viscosity

$\mu_{8} \quad$ solid viscosity

$\nu \quad$ volume fraction of the solid phase

$\nu_{f} \quad$ kinematic viscosity of the fluid

$\pi \quad 3.14159$

$\rho$ mixture density

$\rho_{f} \quad$ fluid (or continuous) phase density

$\rho_{s} \quad$ solid (or distributed) phase density

$\tau \quad$ dummy variable of integration

$\Psi \quad$ free energy density

$\omega \quad$ frequency

$\boldsymbol{\Omega} \quad$ angular velocity of a sphere 


\section{Contents}

1 Introduction 1

2 Single Particle Dynamics 2

2.1 Introduction and Background ................. 2

2.2 Drag . . . . . . . . . . . . . . . . 5

2.3 Lift . . . . . . . . . . . . . ...... 6

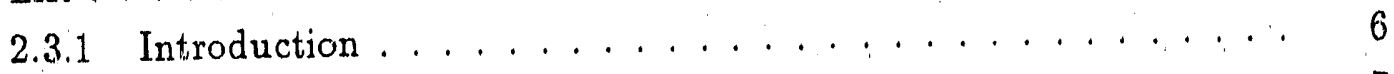

2.3 .2 'Slip-Shear' Lift . . . . . . . . . . . . . . . . . . . . . . . . . . . . . . . . . . . . .

2.3.3 Magnus Force ... . . . . . . . . . . . . 7

2.1. Virtual Mass . . . . . . . . . . . . . . . . . 8

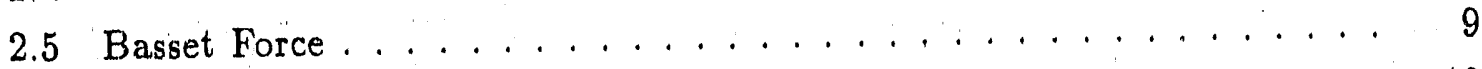

2.6 Faxen Force . . . . . . . . . . . . . . . . . . 10

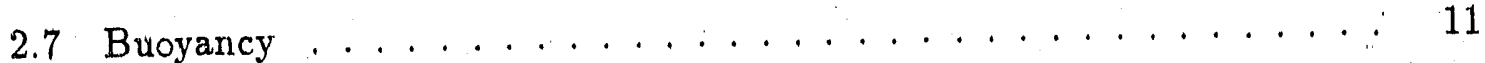

2.8 Pressure Gradient . . . . . . . . . . . . . . . . . . . 11

2.9 Temperature Gradient . . . . . . . . . . . . . . . . . . 11

3 Multicomponent Flows 12

3.1 Introduction and Background . . . . . . . . . . . . 12

3.2 Continuum Theories and Representation of $f_{I} \ldots \ldots \ldots$

3.3 Generalization of Single Particle Results . . . . . . . . . . . . 27

3.3 .1 Drag . . . . . . . . . . . . . . . . . 27

$3.3 .2 \operatorname{Lift} \ldots \ldots \ldots \ldots \ldots \ldots$

3.3.3 Virtual Mass .................... 31

3.3 .4 Basset Force . . . . . . . . . . . . . . . . 33

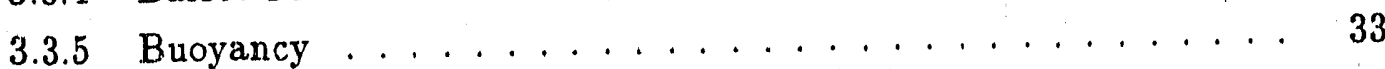

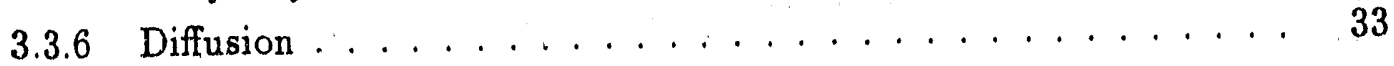

4 Conclusion $\quad 34$

A Frame-Indifference $\quad 38$

A.1 Introduction . . . . . . . . . . . . . . . . . 38

A.2 Change of Frame . . . . . . . . . . . . . . . 39

A.3 Examples ..................... . . 40

B Lift Forces $\quad 42$

B.1 Comparison of 'Slip-Shear' and 'Spin' Lift . . . . . . . . . . . . . 42

B.2 'Ho and Leal' Lift . . . . . . . . . . . . . . . . . . . . . . 44 44 


\section{Introduction}

Multiphase flows have become the subject of considerable attention because of their increasing importance in many industries. The large number of articles published concerning Multiphase flows typically employ one of two continuum theories developed to describe such situations; Mixture Theory (or theory of interacting continua) or Averaging [Ishii, 1975] . Both approaches are based on the underlying assumption that each phase may be mathematically described as a continuum. Mixture theory was first formulated by Truesdell [1957). It provides a means for studying motions of bodies made up of several constituents by generalizing the equations and principles of the mechanics of a single continuum. The fundamental assumption in this theory is that at any instant of time, every point in space is occupied by one particle from each constituent. The historical development and the details of this theory are given in the review articles by Bowen [1976], Atkin and Craine [1976], Bedford and Drumheller [1983], and the recent edition of Rational Thermodynamics [Truesdell, 1984]. In contrast, Averaging directly modifies the classical transport equations to account for discontinuities or 'jump' conditions at moving boundaries between the phases [Drew and Segel, 1971a; Ishii, 1975]. The modified balance equations must then be averaged in either space or time (hence the name Averaging) to arrive at an acceptable local form. In this approach (cf. Anderson and Jackson [1967] and Drew and Segel [1971a]) point-wise equations of motion, valid for a single fluid or a single particle, are modified to account for the presence of the other components and the interactions between components. These equations are then averaged over time or some suitable volume that is large compared with a characteristic dimension (for example, particle spacing or the diameter of solid particles) but small compared to the dimensions of the whole system. Froxn the mathematical manipulation of the averaged quantities, a number of terms (some of unknown physical origin) arise. These terms are usually interpreted as some form of interaction between the constituents. Constitutive relations to represent these interactive forces, as well as for the stress tensors for each constituent, are then required. The primary conceptual difference between the two approaches is the location of the averaging step in the development of the balance equations. Mixture theory implicitly assumes a locally averaged field before any equations are written; Averaging makes it an explicit step in the development of the conservation equations. With few exceptions, both approaches arrive at the same set of equations [Decker, 1988].

Once conservation equations have been established, constitutive relations must be specified to complete the description of the system. Modeling a mixture requires constitutive relations for stress of each component of the mixture and expressions for momentum exchange between the components. Note that this list is for a purely mechanical system (i.e., no temperature differences or chemical reactions are accounted for). In general relations would have to be included for thermal conductivity, chemical reaction rates, etc. (see Bowen [1976]). Selection of appropriate constitutive relations for the stresses in the components of a mixture may be a difficult task, but for our current purpose we assume this has been accomplished. This report considers the selection of constitutive equations describing the momentum exchange, or interaction, between the components of the mixture. Though the continuum theories discussed above do, in 
general, allow one to model a mixture of many components in any flow regime, we will consider only two component mixtures in laminar flow here.

The two-phase flows of concern here typically consist of a large number of solid. particles or gas bubbles suspended in a fluid medium (such as air or water). Most of the momentum exchange relations that have been proposed for these types of twophase flows are based on generalizations of the force balance on a single particle moving through a fluid. The overall plan of this report reflects that approach. First, a discussion and overview of the dynamics of a single particle in a fluid is presented. Then, each of the forces acting on the particle is discussed individually. Finally, continuum theories for representing interaction forces (i.e., momentum exchange mechanisms) are discussed and the expressions for forces on single particles are generalized to mixtures including many particles.

\section{Single Particle Dynamics}

\subsection{Introduction and Background}

Tchen ${ }^{1}$ [1947] synthesizes the work of Basset, Boussinesq, Stokes, and Oseen on the motion of a sphere rettling under the force of gravity in a fluid at rest. The resulting force balance, sometimes known as the Basset-Boussinesq-Oseen (BBO) equation, is given by:

$$
\begin{aligned}
& \frac{4 \pi a^{3}}{3} \rho_{s} \dot{u}=-\frac{2 \pi a^{3}}{3} \rho_{f} \dot{u}-6 \pi \mu_{f} a u \\
& \quad-6 \pi \mu_{f} a \frac{a}{\sqrt{\pi \nu_{f}}} \int_{-\infty}^{t} \frac{\dot{u}\left(t_{1}\right)}{\sqrt{t-t_{1}}} d t_{1}-\frac{4 \pi a^{3}}{3} g\left(\rho_{s}-\rho_{f}\right)
\end{aligned}
$$

where $u$ is the velocity of the particle, $\rho_{f}$ and $\rho_{s}$ are density of the fluid and particle, respectively, $a$ is the particle radius, $g$ is the acceleration of gravity, $\mu_{f}$ and $t^{\prime} f$ are viscosity and kinematic viscosity of the fluid, respectively. The terms on the right hand side of equation 1 reflect the presence of virtual mass, Stokes drag, Basset history effects, and buoyancy. Tchen [1947] modified equation 1 to describe unsteady Stokes motion of a solid spherical particle in a fluid with a uniform flow field. His modifications include replacing the particle velocity by its relative velocity, and the addition of a term accounting for pressure gradients in the fluid. The resulting expression is:

$$
\begin{aligned}
& \frac{4 \pi a^{3}}{3} \rho_{g} \dot{u}=\frac{4 \pi a^{3}}{3} \rho_{f} \dot{v}-\frac{2 \pi a^{3}}{3} \rho_{f}(\dot{u}-\dot{v})-6 \pi \mu_{f} a(u-v) \\
& -6 \pi \mu_{\ddagger} a \frac{a}{\sqrt{\pi \nu_{f}}} \int_{-\infty}^{t} \frac{\dot{u}\left(t_{1}\right)-\dot{v}\left(t_{1}\right)}{\sqrt{t-t_{1}}} d t_{1}-\frac{4 \pi a^{3}}{3} g\left(\rho_{\circ}-\rho_{f}\right)
\end{aligned}
$$

where $v$ is the velocity of the fluid in the neighborhood of the particle but far enough away to be unaffected by it. Note that equation 2 is one scalar component of a more general vector equation. Corrsin and Lumley [1956], noting that equation 2 applies only

\footnotetext{
${ }^{1}$ Tchen's theory is studied extensively by Gouesbet et al. [1982,1984].
} 


\subsection{Introduction and Background}

in the absence of fluid velocity gradients, propose a more general equation, which takes velocity gradients into account in its expression for the pressure gradient. Considering a small rigid sphere of radius $a$ and mass $m$, instantaneously centered at $Y(t)$ and moving with velocity $\mathbf{v}(t)$, they propose the following equation for the force on the sphere:

$$
\begin{aligned}
m_{i} & \frac{d u_{i}}{d t}=\left.m_{f}\left(\frac{D v_{i}}{D t}-\nu_{f} \nabla^{2} v_{i}\right)\right|_{\mathbf{Y}(t)}-\frac{1}{2} m_{f} \frac{d}{d t}\left\{u_{i}(t)-v_{i}[\mathbf{Y}(t), t]\right\} \\
& \quad-6 \pi \mu_{f} \sigma\left\{u_{i}(t)-v_{i}[\mathbf{Y}(t), t]+a \int_{-\infty}^{t} \frac{d \tau \frac{d}{d \tau}\left(u_{i}(\tau)-v_{i}[\mathbf{Y}(\tau), \tau]\right)}{\pi \nu_{f} \sqrt{t-\tau}}\right\} \\
& +\left(m_{\bullet}-m_{f}\right) g_{i}
\end{aligned}
$$

The undisturbed flow field is $v_{i}(\mathbf{x}, t)$ where the subscript $i$ denotes vector component, $m_{f}$ is the mass of fluid displaced by the sphere, and dynamic and kinematic viscosity are $\mu_{f}$ and $\nu_{f}$, respectively. There are two distinct tine derivatives in equation 3 . The derivative $\frac{d}{d t}$ denotes the time derivative following the moving sphere, so that:

$$
\frac{d}{d t} v_{i}[\mathbf{Y}(t), t]=\left.\left(\frac{\partial v_{i}}{\partial t}+u_{j} \frac{\partial v_{i}}{\partial x_{j}}\right)\right|_{\mathbf{X}=\mathbf{Y}(t)}
$$

The derivative $\frac{D}{D t}$ is used to denote the time derivative following a fluid element, and:

$$
\left.\frac{D v_{i}}{D t}\right|_{\mathbf{Y}(t)}=\left.\left(\frac{\partial v_{i}}{\partial t}+v_{j} \frac{\partial v_{i}}{\partial x_{j}}\right)\right|_{\mathbf{X}=\mathbf{Y}(t)}
$$

is the fluid acceleration as observed at the instantaneous center of the sphere.

The source of disagreement over the form of the single particle force balance concerns the pressure gradient term. Tchen [1947] originally proposed the addition of a term accounting for the pressure gradient based on an intuitive argument. He proposed the scalar equation:

$$
f_{p}=V \rho_{f}\left(\frac{\partial v}{\partial t}+v \frac{\partial v}{\partial x}\right)
$$

Corrsin and Lumley [1956] argued that, for a nonuniform flow field, the full NavierStokes equations should be used to determine the pressure gradient (See Section 2.8). They give, in vector form:

$$
\mathbf{f}_{p}=V \rho_{f}\left(\frac{\partial \mathbf{v}}{\partial t}+(\operatorname{grad} \mathbf{v}) \mathbf{v}-\nu \nabla^{2} \mathbf{v}\right)
$$

Buevich [1966] criticizes both previous studies [Tchen, 1947; Corrsin and Lumley, 1956] in pointing out that adding a term to the $\mathrm{BBO}$ equation is not necessary. He uses a change of reference frame to derive a new equation of motion from the original $B B O$ equation and shows that the term:

$$
\mathbf{f}_{p}=V \rho_{f}\left(\frac{\partial \mathbf{v}}{\partial t}+(\operatorname{grad} \mathbf{v}) \mathbf{u}\right)
$$


arises without "the artificial introduction of additionial terms." Soo $[1975,1976)$ argues that the pressure gradient force is exactly balanced by fluid inertia forces and should not appear in the force balance in any form. Maxey and Riley [1983], based on an analysis similar to that of Buevich, incorporate a "pressure" term of the form:

$$
\mathbf{f}_{p}=\left.V \rho_{f}\left(\frac{\partial \mathbf{v}}{\partial t}+(\operatorname{grad} \mathbf{v}) \mathbf{v}\right)\right|_{\mathbf{X}=\mathbf{Y}(t)}
$$

Note that equation 8 incorporates a time derivative following the moving sphere, whereas equation 9 uses a time derivative following a fluid element. Maxey and Riley [1983] argue that equation 9 is the more physically realistic expression in that the effect of stresses caused by pressure and viscosity is to produce the same net force that would act on a fluid sphere of the same size. They propose the following equation for the force on a sphere in a nonuniform flow:

$$
\begin{aligned}
& m_{a} \frac{d u_{i}}{d t}=\left(m_{s}-m_{f}\right) g_{i}+\left.m_{f} \frac{D v_{i}}{D t}\right|_{\mathbf{Y}(t)} \\
& -\frac{1}{2} m_{f} \frac{d}{d t}\left\{u_{i}(t)-v_{i}[\mathbf{Y}(t), t]-\left.\frac{1}{10} a^{2} \nabla^{2} v_{i}\right|_{\mathbf{Y}(t)}\right\} \\
& -6 \pi a \mu_{f}\left\{u_{i}(t)-v_{i}[\mathbf{Y}(t), t]-\frac{1}{6} a^{2} \nabla^{2} v_{i} \mid \mathbf{Y}(t)\right\} \\
& +6 \pi a^{2} \mu_{f} \int_{0}^{t} d \tau\left(\frac{\frac{d}{d \tau}\left\{u_{i}(\tau)-v_{i}[\mathbf{Y}(\tau), \tau]-\frac{1}{6} a^{2} \nabla^{2} v_{i} \mid \mathbf{Y}(\tau)\right\}}{\sqrt{\pi \nu_{f}(t-\tau)}}\right)
\end{aligned}
$$

Note that the inclusion of velocity gradients in their analysis results in modifications to the virtual mass, Stokes drag, and Basset history terrns. These velocity gradients correspond to the physical effect known as Faxen forces and will be discussed later.

\begin{tabular}{||l|l|}
\hline \multicolumn{2}{|c|}{ Table 1. Force Due to Pressure Gradient } \\
\hline \hline Author & Pressure Term \\
\hline Tchen, 1947 & $V \rho_{f}\left(\frac{\partial v}{\partial t}+v \frac{\partial v}{\partial \mathbf{w}}\right)$ \\
Corrsin and Lurnley, 1956 & $V \rho_{f}\left(\frac{\partial \mathbf{v}}{\partial t}+(\operatorname{grad} \mathbf{v}) \mathbf{v}-\nu \nabla^{2} \mathbf{v}\right)$ \\
Buevich, 1966 & $V \rho_{f}\left(\frac{\partial \mathbf{v}}{\partial t}+(\operatorname{grad} \mathbf{v}) \mathbf{u}\right)$ \\
Soo, 1975,1976 & 0 \\
Maxey and Riley, 1983 & $\left.V \rho_{f}\left(\frac{\partial \mathbf{v}}{\partial t}+(\operatorname{grad} \mathbf{v}) \mathbf{v}\right)\right|_{\mathbf{X}=\mathbf{Y}(t)}$ \\
\hline
\end{tabular}


Though equation 10 is complete for a single particle in Stokes flow, there are, in general, other forces that must be considered (even for a purely mechanical system). In flows with high relative velocity between phases, or large velocity gradients in the fluid phase, lift may become an important effect [McLaughlin, 1989]. Also, spin of the particle is not taken into account in the above equations. Each of the effects included in equation 10, plus these two that are not, will now be discussed individually. ${ }^{2}{ }^{3}$

\subsection{Drag}

At low Reynolds number (i.e., low relative velocity) the inertial terms in the NavierStokes equations may be neglected. The resulting drag force on a sphere is given by [Stokes, 1851] :

$$
f_{D}=6 \pi a \mu_{f} v_{r}
$$

where $\mu_{f}$ is the viscosity of the fluid and all other variables are defined as above. Equation 11 is known as Stokes' law for the resistance of a moving sphere.

Forces exerted on bodies moving through a fluid are generally expressed in terms of a dimensionless drag coefficient, $C_{D}$, defined through the relation:

$$
C_{D}=\frac{f_{D}}{\frac{1}{2} \rho_{f} v_{r}^{2} \pi a^{2}}
$$

With this definition, Stokes' law (equation 11) yields:

$$
C_{D}=\frac{24}{R e}
$$

where:

$$
R e=\frac{2 a v_{\tau} \rho_{f}}{\mu_{f}}
$$

is the Reynolds number based on the sphere diameter. This drag coefficient is generally an acceptable approximation up to $R e=0.2$ [Khan and Richardson, 1987]. Oseen modified Stokes' solution by including the inertial terms of the Navier-Stokes squations for the flow field far from the body [Schlichting, 1979]. The Oseen improvernent is given by:

$$
C_{D}=\frac{24}{R e}\left(1+\frac{3}{16} R e\right)
$$

and is applicable for values of Reynolds number up to about 2. If $R e \geq 2$, the usual approach is to determine $C_{D}$ experimentally as a function of particle Reynolds number and present it as a table, graph, or correlation. Morsi and Alexander [1972] present a theoretical investigation of the flow of a spherical particle (i) in a one-dimensional flow, (ii) in a uniform two-dimensional fluid flow about a circular cylinder, and (iii) about a

\footnotetext{
${ }^{2}$ Robinson [1956] also studies the motion of small particles in a potential field of flow. Rizk and Elghobashi [1985] study the motion of a spherical particle in a turbulent flow near a wall.

${ }^{3}$ The behavior of bubbles, drops and particles in various flow fields is discussed in the book by Clift et al. [1978].
} 
lifting aerofoil section. The experimental drag curve is divided into a number of regions and is approximated in each region by a curve of the form:

$$
C_{d}=\frac{k_{1}}{R e}+\frac{k_{2}}{R e^{2}}+k_{3}
$$

where the values of $k_{1}, k_{2}$, and $k_{3}$ are given for different ranges of the Reynoids number in the Appendix of Morsi and Alexander [1972]. Khan and Richardson [1.987] present an extensive review and critique of available experimental data and correlations for drag on a single sphere.

\subsection{Lift}

\subsubsection{Introduction}

It is observed [Segre and Silberberg, 1961; Segre and Silberberg, 1962a,b] that spheres in laminar Foiseuille flow through a pipe (at low Re) accumulate in an annulus some distance from the tube axis. Following the initial observations, a number of investigators verify this 'tubular pinch' effect and attempt to explain the lateral (or lift) fnrce acting on the spheres [Denson et al., 19606; Eichorn and Small, 1964; Jeffrey and Pearson, 1965; Oliver, 1962; Repetti and Leonard, 1964]. Though some authors attempt to explain the radial migrations in terms of particle spin (i.e., Magnus forces), spheres prevented from spinning ${ }^{4}$ also reached equilibrium positions between wall and centerline [Denson et al., 1966; Lawyer and Lu, 1971; Oliver, 1962; Repetti and Leonard, 1964]. Lawyer and $\mathrm{Lu}^{5}$ [1971] and Brenner [1966] give detailed reviews and comparisons of these experimental results. Denson et al. [1966] and Aoki et al. [1979] both fail to observe annulus formation at particle Reynolds numbers higher than those used by the previous investigators. They do, however, agree with previous results in that particles lagging the flow migrate to the tube axis and particles preceding the flow migrate towards the tube wall.

Bretherton [1962] shows that, based on creeping flow equations, there is no lateral force on a single rigid spherical particle in a unidirectional flow. Saffman $[1965,1968]$ deduces that, since experimental results contradict this conclusion, inertial effects must be involved. Saffman sbtains a result for 'slip-shear' lift on a particle at low Reynolds number analogous to a result derived earlier for 'spin' lift by Rubinow and Keller [1.961]. Both results are discussed in the following sections. Saffman includes particle spin in his analysis and shows [Saffman, 1965] that, under circumstances where his results and Rubinow and Keller's results strictly apply, the 'shear' lift dominates the 'spin' lift (see also Boothroyd [1967] ). Aoki et al. [1979] claims that for larger particle Reynolds numbers (i.e. $R e_{p} \geq 1$ ) the Magnus effect explains the observed particle migrations. Here, the particle Reynolds number, $R e_{p}$, is defined as:

$$
R e_{p}=\frac{a v_{r} \rho_{f}}{\mu_{f}}
$$

\footnotetext{
${ }^{4}$ One author [Oliver, 1962] drills a small hole in the side of each sphere so that the offset center of gravity counteracts rotation.

${ }^{5}$ Lawyer and Lu [1971], after reviewing previous particle migration results, calculate particle trajectories using an incorrect expression for Saffman's [Saffman, 1965] 'slip-shear' lift.
} 
Ho and Leal [1974] calculate another form of lift force on a single particle in a channel. This force is apparently a result of wall effects and is discussed in Appendix B. Auton [1987] studies the lift force on a sphere ciue to a weak shear flow of an inviscid fluid.

\subsection{2 'Slip-Shear' Lift}

Saffman $[1965,1968]$ uses an expansion valid for small values of the reciprocal viscosity to calculate the lift force on a small sphere in a slow unbounded simple shear flow. His result is given by:

$$
f_{L}=6.46 \mu_{f}^{\frac{1}{2}} \rho_{f}^{\frac{1}{2}} a^{2} k^{\frac{1}{2}}(u-v)
$$

where $k$ is the velocity gradient in simple shear, $\rho_{f}$ the density of the fluid, $a$ is the radius of the sphere, $u$ is the particle velocity and $v$ is the fluid velocity. Even though Saffman has retained the inertial terms of the Navier-Stokes equations in his analysis, the flow is not inertially dominated. Decker [1988] points out that Saffman's analysis of lift force includes the following implicit assumptions: the flow is uniform and parallel, the slip velocity is parallel to the plane of fluid shear, the shear or velocity gradients of the fluid are linear and the particle spin vector lies in the plane of fluid shear, but is normal to the slip vector. The lift force Saffman derives is normal to the slip vector and the spin vector of the particle. If the particle lags the fluid, the lift will move the particle towards the faster adjacent fluid and vice versa if the particle leads the fluid.

\subsubsection{Magnus Force}

Consider a spinning bocy traveling through a fluid such that its axis of rotation does not coincide with its direction of translation (i.e., its path or trajectory). The body will experience a lift force in a direction perpendicular to the plane defined by its axis of rotation and its trajectory. The magnitude of this force, known as the Magnus force, depends upon spin rate, velocity, and shape of the body. The so-called 'Magnus effect,' in which the force just described causes a deflection perpendicular to the flight path, was first investigated in relation to deflection of projectiles (such as tennis balls or baseballs). Although named after Magnus, who studied the lift caused by rotation of a cylinder, the effect of spin on the flight of tennis balls was first noted by Newton in the 1600s. An historical review is given by Barkla and Auchterlonie [1971] .

A relatively large amount of work has been done concerning the Magnus force acting on spinning objects of large size [Swanson, 1961]. The theoretical analyses for these situations, however, are based on inviscid flow and are therefore inappropriate for predicting lift on small particles at low Reynolds numbers where viscous effects are expected to dominate. Generally, lift coefficients are measured empirically; though ex. perimental work has also been primarily concerned with large objects at high Reynolds numbers. Tsuji et al. [1985] performs experiments on small spheres ( $5 \mathrm{~mm}$ dia.) at, Reynolds numbers as low as 550 . These are apparently the smallest particles and lowest Reynolds numbers for which experimental data exists.

Rubinow and Keller [1961] obtain an analytic expression for the lift force on a small spinning sphere. They used an expansion valid for small values of the particle 
Reynolds number to calculate the transverse force on the sphere. For a sphere of radius $a$, spinning with angular velocity $\Omega$, and moving through an unbounded stationary fluid with velocity $\mathbf{u}$, they obtain a lift force of:

$$
\mathbf{f}_{L}=\pi \rho_{f} a^{3} \mathbf{\Omega} \times \mathbf{u}
$$

where $\rho_{f}$ is the density of the fluid. Unfortunately, this is a rather restricted result and attempts to extend the expansion technique to situations where there are velocity gradients in the fluid (such as in Poiseuille flow) have failed. Jeffrey and Pearson [1965] attempt a semi-empirical comparison of their experimental results for particles in Poiseiulle flow with Rubinow and Keller's equation. Though the results of the comparison are not convincing, the r-dependence of the calculated and measured radial velocities corresponds reasonably well.

Clearly, the Magnus effect can be quite important when the body is 'spun' by an external force. It is not clear, however, what spin, if any, a particle in an arbitrary flow might have. Unless it has just struck a bounding wall or some other obstacle, one might expect the particle to have a spin equal to the vorticity of the fluid velocity field, or even no spin at all. For a sphere in' a Poiseuille flow, Jeffrey and Pearson [1965] observe experimentally that:

$$
\Omega=\frac{1}{2} \operatorname{curl} \mathrm{v}
$$

to within $7 \%$ for neutrally buoyant spheres, and to within their experimental error for spheres with a higher density.

\subsection{Virtual Mass}

When a particle is accelerating with respect to a fluid, an added mass force, which is not present under steady conditions, must be included in the force balance on the particle. A good qualitative explanation is given by Birkhoff [1960] :

... let a light paddle be dipped into still water and then suddenly given a rapid acceleration broadside. It is a matter of common experience that the apparent inertia (i.e., resistance to acceleration) of the paddle is greatly increased by the water around it. This increased inertia is what is called the "virtual mass" of the paddle, the difference between the real mass and the virtual mass being called the "induced mass" or "added mass."

The virtual mass is a means of quaritifying the force required to displace the fluid surrounding the accelerating body. One can write Newton's Second Law for a solid spherical body of diameter $D$ and density $\rho_{A}$, accelerating (at a) through a liquid of density $\rho_{f}$, as [Lahey, 1982] :

$$
\mathbf{f}_{s}=\left(m_{s}+m_{v m}\right) \mathbf{a}_{v m}=\frac{4 \pi}{3} a^{3}\left(\rho_{s}+\frac{1}{2} \rho_{f}\right) \mathbf{a}_{v m}
$$

The added mass, $m_{v m}$, accounts for having to accelerate the surrounding liquid. $m_{v m}$ is equal to one-half the mass of the displaced liquid for a perfect sphere. The virtual 
mass coefficient, $C_{v m}$ is defined through:

$$
\mathbf{f}_{v m}=C_{v m} \rho_{f} \mathbf{a}_{v m}
$$

where $C_{v m}=1 / 2$ for a single perfect sphere accelerating through a fluid medium and $\mathbf{a}_{v m}$ is an expression for the particle acceleration. Clearly, from equation 21, virtual mass has greater relative importance in the force balance when the particle has a lower density in comparison to the fluid. ${ }^{6}$

\subsection{Basset Force}

A particle accelerating through a fluid medium will experience virtual mass and Basset forces. One may think of virtual mass forces as resulting from the potential flow field created by the accelerating particle. Likewise, the Basset force may be considered n result of the viscous flow field created by the particles motion. Viscous phenomena, such as boundary layer growth, are described by diffusion equations and thus the flow field at time $t$ is a function of the entire history of the particle motion. For laminar flow around a sphere, Basset [1888] obtained the result:

$$
\mathbf{f}_{H}=-6 \rho_{f} a^{2} \sqrt{\pi \nu_{f}} \int_{t_{o}}^{t}\left(t-t^{\prime}\right)^{-\frac{1}{2}} \frac{d}{d t^{\prime}}(\mathbf{u}-\mathbf{v}) d t^{\prime}
$$

where $\rho_{f}$ is the fluid density, $d$ is the particle diameter, $\mathbf{u}$ and $\mathbf{v}$ are the velocity vectors, $t$ is time, $t^{\prime}$ is the dummy variable of integration, and $\nu_{f}$ is the kinematic viscosity. This expression constitutes an instantaneous flow resistance and may be considered a correction to the viscous drag accounting for transient conditions; it is often referred to as the Basset integral. It has certain limitations in that it was derived for the situation of a particle experiencing high acceleration at low velocity.

Ahmadi and Goldschmidt [1971] showed that the Basset integral does not have a strong effect on particle motion at sufficiently long times (large $t$ ). It may, howevcr, contribute significantly at short times and several studies of a sphere movirig in a fluid have incorporated Basset forces. Odar and Hamilton [1964] attempt to extend Basset's result to higher velocities. They rewrite equation 23 in a more general form:

$$
\mathbf{f}_{H}=-C_{H} \rho_{f} a^{2} \sqrt{\pi \nu_{f}} \int_{t_{o}}^{t}\left(t-t^{\prime}\right)^{-\frac{1}{2}} \frac{d}{d t^{\prime}}(\mathbf{u}-\mathbf{v}) d t^{\prime}
$$

where all the previous variables have the same definitions, $a$ is the particle radius, and $C_{H}$ is a numerical coefficient to be determined. They also define the acceleration number as:

$$
A c=\frac{u^{2}}{2 a u_{t}}
$$

where $u, a$, and $u_{t}$ are velocity, radius, and local acceleration of the sphere, respectively. By performing experiments with a sphere oscillating in a fluid the authors obtain empirical values of $C_{H}$ for $0<A c<2$. Odar [1966] later finds that the values of $C_{H}$

\footnotetext{
${ }^{6}$ Kowe et al. [1988) analyzes the motion of a single bubble or particle in an acelerating fluid flow where the effect of virtual rnass becornes important.
} 
measured for the oscillating sphere discussed above also predict free fall of a sphere quite well. He then expresses the data from Odar and Hamilton's previous paper by the following correlation:

$$
C_{H}=2.88+\frac{3.12}{(A c+1)^{3}}
$$

Note that as $v \rightarrow 0$ or $a \rightarrow \infty, A c \rightarrow 0$ and $C_{H} \rightarrow 6$ which is the value Basset obtained.

Hjelmfelt et al. [1967] derives and solves the equation of motion describing a sphere oscillating in a fluid. Obtaining displacement as a function of time, they show that the Basset history integral contributes significantly to the calculated displacement of the sphere, and in some situations may be more important than virtual mass or drag. Hjelmfelt and Mockros [1967] perform a similar study for spheres falling in a viscous fluid. Again, Basset's history integral is shown to be quite important. In the above study, the displacement-time relations for the falling spheres are verified by experiment. See also A hmadi and Goldschmidt [1971].

It is apparent from equation 24 that $\mathrm{f}_{H}$ becomes large when the local acceleration is high. This fact is demonstrated experimentally by Hughes and Gilliland [1952] who observe a drag force many times that due to steady state drag. It is not as apparent from equation 24 (which represents a single term in the equation of motion of the sphere) that $f_{H}$ is more important for greater values of $\rho_{f} / \rho_{s}$. For small values of this density ratio, the Basset term may generally be neglected [Soo, 1967] ${ }^{7}$.

Tatem [1988] has recently shown that the Basset integral may be expressed as a semi-derivative $e^{8}$. Though Tatem is simply presenting a different way to write the same quantity, it may simplify numerical calculations to replace the Basset integral with his expression.

\subsection{Faxen Force}

Stokes' expression for drag is derived for a single sphere translating at low Reynolds number in a stationary fluid. The drag force on a sphere translating through a fluid that is moving and has velocity gradients will generally have a different value. For steady Stokes flow, the correction for this condition is given by the Faxen relations [Happel and Brenner, 1973], so that the drag force on a sphere is:

$$
\mathbf{f}_{F}=6 \pi a \mu_{f}(\mathbf{v}-\mathbf{u})+\mu_{f} \pi a^{3} \nabla^{2} \mathbf{v}
$$

where $a$ is the sphere radius, $\mu_{f}$ is the viscosity of the fluid, $u$ is the velocity of the sphere, and $\mathbf{v}$ is the velocity of the fluid. Including this effect in the force balance on a sphere results in modifications (due to the curvature of the velocity profile) to the virtual mass term and the Basset history term in addition to the Stokes drag [Maxey and Riley, 1983] .

\footnotetext{
${ }^{7}$ In some applications small particles and droplets may encounter normal shocks. Forney et al. [1987] studies the effect of the Basset history integral behind a normal shek wave. The effect of Basset forces on asymptotic particle turbulent diffusivity is studied by Shi [1987]

${ }^{8}$ By semi-derivative Tatem means a fractional derivative "according to the Riemann-Liouville integral definition. ${ }^{n}$
} 


\subsection{Buoyancy}

When body forces are present, a particle immersed in a fluid medium of different density than the particle will experience a buoyancy force. Evaluating the magnitude of this force is a simple matter that is discussed in any elementary book on fluid dynamics (for example White [1986]). Consider a sphere immersed in a fluid medium with the entire system in a gravity field. Balancing forces on the sphere gives:

$$
f_{B}=\rho_{s} V_{a} g+V_{A} \rho_{f} g-\left(V_{A}+V_{a}\right) \rho_{f} g
$$

where $f_{B}$ is the net buoyancy force, $\rho_{0}$ is the density of the sphere, $\rho_{f}$ is density of the fluid, $V_{a}$ is the volume of the sphere, $V_{A}$ is the volume of the fluid above the sphere, and $g$ is the acceleration of gravity. This equation can be simplified to:

$$
f_{B}=V_{s} g\left(\rho_{s}-\rho_{f}\right)=g\left(m_{s}-m_{f}\right)
$$

where $m_{6}$ and $m_{f}$ are the mass of the sphere and the mass of an equal volume of fluid, respectively.

\subsection{Pressure Gradient}

An object of finite size immersed in a viscous fluid with a pressure gradient will experience a force due to that pressure gradient. This force arises from the fact that the pressure on the 'upstream' side of the object will be greater than that on the 'downstream' side. A force balance on a sphere in a fluid with a pressure gradient yields the relation:

$$
\mathbf{f}_{p}=-V_{s} \nabla p_{f}
$$

The Navier-Stokes equation (without body forces) may be written:

$$
-\frac{1}{\rho_{f}} \nabla p_{f}=\frac{D \mathbf{v}}{D t}-\nu_{f} \nabla^{2} \mathbf{v}
$$

so that equation 30 may be rewritten as:

$$
\mathfrak{f}_{p}=V_{\iota} \rho_{f}\left(\frac{D \mathbf{v}}{D t}-\nu_{f} \nabla^{2} \mathbf{v}\right)
$$

or, defining:

$$
m_{f}=V_{\imath} \rho_{f}
$$

as:

$$
\mathbf{f}_{p}=m_{f}\left(\frac{D \mathbf{v}}{D t}-\nu_{f} \nabla^{2} \mathbf{v}\right)
$$

\subsection{Temperature Gradient}

When a small particle or a liquid drop is suspended or inserted in a gas or a liquid which is not in thermal equilibrium, i.e., a temperature gradient exists, then the particle moves in the direction of decreasing temperature (if there are no other outside forces acting on the particle). ' $\mathrm{t}$ his phenomenon is studied by Phillips [1975], Talbot et al. [1980], Rosner [1988], Barton and Subramanian [1989], and Kempers [1989] among others. 


\section{Multicomponent Flows}

\subsection{Introduction and Background}

In proposing the constitutive relation for the diffusive body force (i.e., interaction force) in a multiparticle system, many investigators (Anderson and Jackson, 1967; Drew and Segel, 1971a; Homsy et al., 1980] generalize the problem of a single spherical particle undergoing slow rectilinear motion in an inflnite fluid by introducing a void fraction dependence for certain coefficients. The interactive force is, in general, a function of the fluid pressure gradient; the density gradients (the buoyancy forces); the relative velocity (the drag force on the particles); the relative acceleration (the virtual mass of the particles); the magnitude of the rate of deformation tensor of the fluid ( the lift force on the particles); the spinning motion, as well as the translation of particles (the Faxen's force); the particles' tendency to move toward the region of higher velocity (the Magnus effect); the history of the particle motion (the Basset force); etc.

Conformance with the accepted principles of constitutive equations as given by Truesdell and Noll [1965], such as invariance to coordinate transformations, frame indifference, homogeneity, and isotropy, is essential in forming constitutive models for two-phase continua (see Appendix A).

In this section of the report, we will present an historical overview of the development of expressions for the interaction forces in multicomponent flows. The following sections will concentrate on continuum theories for the representation of the interaction force and generalization of the single particle results already discussed to the case of many particles.

In generalizing the forces acting on a single particle to a mixture of solid particles and fluids, many investigators start with the work of Landau and Lifshitz [1959]. They show that the velocity of a single sphere, being an arbitrary function $u(t)$, can be represented as a Fourier integral:

$$
u(t)=\int_{-\infty}^{\infty} u_{\omega} e^{-i \omega t} d \omega
$$

or

$$
u_{\omega}=\frac{1}{2 \pi} \int_{-\infty}^{\infty} u(\tau) e^{i \omega t} d \tau
$$

Since, assuming creeping flow, the equations are linear, the total drag may be written as the integral of the drag forces for velocities that are the separate Fourier components $u_{w} e^{-i \omega t}$. These forces are given in Landau and Lifshitz [1959] as:

$$
\pi \rho_{f} a^{3} u_{\omega} e^{-i \omega t}\left\{\frac{6 \nu_{f}}{a^{2}}-\frac{2 i \omega}{3}+\frac{3 \sqrt{2 \nu_{f}}}{a}(1-i) \sqrt{\omega}\right\} .
$$

Noticing that:

$$
\left(\frac{d u}{d t}\right)_{\omega}=-i \omega u_{\omega}
$$

we can write the above as:

$$
\pi \rho_{f} a^{3} e^{-i \omega t}\left\{\frac{6 \nu_{f}}{a^{2}} u_{\omega}-\frac{2}{3}(\dot{u})_{\omega}+\frac{3 \sqrt{2 \nu_{f}}}{a}(\dot{u})_{\omega} \frac{(1+i)}{\sqrt{\omega}}\right\} .
$$


Integrating over $\omega$, the first and second terms give $u(t)$ and $\dot{u}(t)$, respectively. The somewhat more complicated integration of the third term is shown in Landau and Lifschitz [1959]. It is shown that:

$$
2 \operatorname{Re}\left\{(1+i) \int_{0}^{\infty} \frac{(\dot{u})_{\omega}}{\sqrt{\omega}} e^{-i \omega t} d \omega\right\}=\frac{2}{\pi} \int_{-\infty}^{t} \frac{\dot{u}(\tau)}{\sqrt{t-\tau}} d \tau
$$

Thus, we can finally write the expression for the drag as:

$$
f_{D}=2 \pi_{f} \rho_{f} a^{3}\left\{\frac{1}{3} \frac{d u}{d t}+\frac{3 \nu_{f} u}{a^{2}}+\frac{3 \nu_{f}}{a \pi} \int_{-\infty}^{t} \frac{d u}{d \tau} \frac{d \tau}{\sqrt{t-\tau}}\right\}
$$

or:

$$
f_{D}=\frac{2}{3} \pi \rho_{f} a^{3} \frac{d u}{d t}+6 \pi \mu_{f} a u+6 \rho_{f} a^{2} \sqrt{\rho_{f} \mu_{f} \pi} \int_{-\infty}^{t} \frac{d u}{d \tau} \frac{d \tau}{\sqrt{t-\tau}}
$$

In general, equation 42 can be rewritten as:

$$
\mathbf{f}_{D}=\alpha_{1} \mathbf{u}+\alpha_{2} \frac{d \mathbf{u}}{d t}+\alpha_{3} \int_{-\infty}^{t} \frac{d \mathbf{u}}{d 1} \frac{d \tau}{\sqrt{t-\tau}}
$$

where:

$$
\begin{gathered}
\alpha_{1}=6 \pi \mu_{f} a \\
\alpha_{2}=\frac{2}{3} \pi \rho_{f} a^{3} \\
\alpha_{3}=6 \rho_{f} a^{2} \sqrt{\rho_{f} \mu_{f} \pi}
\end{gathered}
$$

If, in addition to the particle velocity $\mathbf{u}$, the fluid is also moving with velocity $\mathbf{v}$, then to generalize equation 43 we need only replace $\mathbf{u}$ by the relative velocity $(\mathbf{u}-\mathbf{v})$.

Anderson and Jackson [1967] appear to be the first who motivate the use of an interaction force of type 43 . Their reasoning for using equation 43 is that the interaction force shoula depend "rot only on the instantaneous motion of the particle, but also on the instantaneous fluid velocity field in which it moves and which in turn depends on the complete history of the particle's motion." They, however, neglect the effects of the last term in equation 43 , which is usually referred to as the "Basset force".

When equation 43 is generalized to the case of an assembly of particles, it seems natural to assume that the coefficients $\alpha_{1}, \alpha_{2}$, and $\alpha_{3}$ become functions of the void fraction $\varepsilon$. This is indeed suggested by Anderson and Jackson [1967], who further comment:

It seems less reasonable, however, to include a term analogous to the Basset force, since the presence of a large assembly of particles dispersed throughout the fluid is likely to erase any historical effect of the motion of a given particle on the fluid flow in its own neighborhood. 
Anderson and Jackson [Anderson and Jackson, 1967] and Jackson [Jackson, 1970; Jackson, 1971; Jackson, 1985], in a series of papers, use different versions of 43 in their analysis, e.g.:

$$
\mathbf{f}_{I}=\varepsilon \beta(\varepsilon)(\mathbf{u}-\mathbf{v})+(1-\varepsilon) C(\varepsilon) \rho_{f} \frac{D}{D t}(\mathbf{u}-\mathbf{v})+(1-\varepsilon) \operatorname{div} \mathbf{T}_{f}
$$

where $u$ and $v$ are the velocities of the solid and fluid constituents respectively, $\varepsilon(\mathbf{x}, t)$ is the voidage fraction of volume occupied by the fluid phase, $\beta(\varepsilon)$ is the drag coefficient, and $C(\varepsilon)$ is the virtual mass coefficient. According to these authors, last term in this equation accounts for the presence of the buoyancy force. Another form used by Jackson [Jackson, 1985] is:

$$
\mathbf{f}_{I}=\beta(\varepsilon,|\mathbf{u}-\mathbf{v}|)(\mathbf{u}-\mathbf{v})+\left(\frac{1-\varepsilon}{\varepsilon}\right) C(\varepsilon) \rho_{f} \frac{D}{D t}(\mathbf{u}-\mathbf{v}) .
$$

The coefficient of drag, $\beta(\varepsilon)$, is studied extensively and will be discussed later in this report. Anderson and Jackson, for lack of any experimental data or physical observation, use a value of one-half for $C(\varepsilon)$ in their analyses. This is the value for the virtual mass coefficient when a single sphere is moving through an infinite fluid. An important shortcoming in the works of Anderson and Jackson [1967, 1968] and of Jackson [1971] is the use of frame-dependent forms for the relative acceleration. This is a common misunderstanding among workers in the area of two-phase flow [Murray, 1965; Soo, 1967; Wallis, 1969] and is discussed later in this report and in the appendix.

Drew and Segel [1971a] consider a structure for the interactive force similar to equation 45 , but do not include the $\operatorname{div} T_{f}$ in their equation. Instead, they consider the effect of surface tension and also include a term that represents the force on the particles due to the pressure of the fluid phase. They show that this term is related to the gradient of the volume fraction of the solid particles and contains the buoyancy forces. The form specifically proposed by them for the interactive force is:

$$
\begin{aligned}
\mathbf{f}_{I}= & -p_{f} \operatorname{grad} \alpha^{*}+\mathbf{S}(\mathbf{u}-\mathbf{v}) \\
& +\mathbf{M}\left\{\left[\frac{\partial \mathbf{u}}{\partial t}+\operatorname{grad} \mathbf{u}(\mathbf{u}-\mathbf{v})\right]-\left[\frac{\partial \mathbf{v}}{\partial t}+\operatorname{grad} \mathbf{v}(\mathbf{v}-\mathbf{u})\right]\right\}
\end{aligned}
$$

where:

$$
\mathbf{f}_{I}+\mathbf{f}_{t}=\mathbf{T}^{*} \operatorname{grad} \mathbf{A}
$$

where $T^{*}$ is the average surface tension tensor, $\mathbf{A}$ depends on the local geometry, $\alpha^{*}$ is related to the void fraction, $S$ is called the drag coefficient tensor, and $M$ is the virtual mass tensor.

The appearance of the first term $\left(-p_{f} g r a d \alpha^{*}\right)$ in equation 47 is of fundamental importance in multicomponent flows. Drew and Segel [1971a] arrive at this conclusion by assuming that the interactive force has a component due to the fluid pressure (with further assistance from their averaging technique). Nevertheless, the inclusion of density gradients in nostulating constitutive equations for the interactive force is a milestone in the theory of mixtures (due to Müller [1968]) ${ }^{8}$.

\footnotetext{
${ }^{9}$ The reason for including the gradient of the densities in the diffusive body force is that, in a
} 


\subsection{Continuum Theories and Representation of $\mathrm{f}_{I}$}

When describing, from a continuum point of view, the behavior of materials such as rubber, water, metals, or polymers, one can regard each of these as a single continuum. However, in many instances, we encounter ideal gas mixtures, fluid mixtures, bubbly liquids, alloys, suspensions, fluidized particles, porous media and pneumatic systems that cannot be regarded as a single constituent continuum. It is more appropriate to assume that the material consists of more than one constitnent. A general theory of interacting continua, i.e., mixture theory, based on modern continuum mechanics can be applied to the above-mentioned problems. Truesdell [195'] is the first to set up the mathematical theory of mixtures in which phenomena of diffusion, dissociation, combination, and chemical reaction in the broadest sense can be represented. Later, Truesclell [1962] studies the mathematical theory of the diffusion of mass in a mixture using four different approaches; namely: (i) the kinematical, leading to Fick's equation of diffusion, (ii) the hydrodynamical (Maxwell-Stefan equations of motion for the constituents in a mixture of fluids), (iii) the kinetic (Maxwell-Chapman-Enskog formulas in a mixture of dilute monatomic gases), and (iv) the thermodynamic approach, which is suitable for the diffusive flux in more general fluid mixtures.

Adkins [1963a,b] develops a theory of non-linear diffusion based on ideas of Truesdell and Toupin [1960), i.e., mixture theory. To account for diffusion phenomena, Adkins $[1963 \mathrm{a}, \mathrm{b}]$ assumes that the body force acting on a given component (per unit mass of that component) can be subdivided into an extraneous body force that is identical in character for single component systems, and a diffusive force. Adkins [1963a] assumes that this diffusive force depends upon the densities of the constituents at the point under consideration and upon their relative velocities. In a follow-up paper, Adkins [1963b] presents a generalization that makes the diffusive forces dependent, in addition, upon velocity gradients and their time derivatives (related to virtual mass effects), and also upca density gradients. In both cases, the form of the force is restricted to satisfy the necessary requirements for invariance under rigid body motions (principle of material frame-indifference). Adkins $[1963 \mathrm{a}, \mathrm{b}]$ studies a few problems such as steady

fluid-solid system, the particles need not be uniformly distributed. Müller [1968] gives an interesting thermodynamical argument for the inclusion of the deneity gradients. He showed that the omission of the density gradients would lead to models that are too restrictive. He called such mixtures "simple" and he found that for a simple mixture of two fluids:

$$
\rho \Psi_{I}=\rho_{1} \Psi_{1}\left(\theta, \rho_{1}\right)+\rho_{2} \Psi_{2}\left(\theta, \rho_{2}\right)
$$

where $\Psi_{1}$ and $\Psi_{2}$ are the free energy densities for the two constituents and $\rho_{1}$ and $\rho_{2}$ are the densities of the two constituents measured per unit volume of the mixture.

The result that $\Psi_{1}$ is independent of $\rho_{2}$ and $\Psi_{2}$ is independent of $\rho_{1}$ are known from classical thermochemistry to be too special. Müller [1968] showed that a mixture of ideal gases is a simple mixture. However, in general, such a conditinn on the free energy is too rostrictive and does not include most fluid mixtures. Therefore, to avoid such a restrictive theory, inclusion of density gradients in the constitutive equations appears necessary. Als , in hydrodynamics a body submerged in a fluid experiences a force proportional to the density difference between the body and the fluid. Similarly, Müller [1968] reasons that, if one visualizes the mixture as a multitude of molecules in which the molecules in the $\alpha$ phase are distributed uniformly but the molecules of the other phase are not, then there is a resultant force upon the $\alpha$-molecules due to intermolecular interaction. 
diffusion through fluid in laminar flow, flow of mixture between rotating cylinders, propagation of plane waves through a mixture, etc., using this theory. Through a series of assumptions and a very interesting analysis, Adkins [1963b] shows that the classical diffusion equations based on Fick's law can be obtained using his non-linear theory.

In their theory of non-linear diffusion, Green and Adkins [1964] regard the diffusive force $\Psi$ (which is equal to $\frac{\Gamma_{1}}{\rho_{1} \rho_{3}}$ in our notation) as "a retarding force exerted on each fluid due to the other and it is natural to suppose that it at least depends on all the quantities that appear in the constitutive equations for the partial stresses..." They therefore assume that:

$$
\Psi=\Psi\left({ }^{1} \mathrm{D},{ }^{2} \mathrm{D}, \boldsymbol{\Gamma}-\boldsymbol{\Lambda}, \mathbf{u}-\mathbf{v}, \rho_{1}, \rho_{2}\right)
$$

where ${ }^{1} \mathrm{D}$ and ${ }^{2} \mathrm{D}$ are the strain rate tensors for two phases, and $\Gamma$ and $\Lambda$ are the skewsymmetric parts of the velocity gradients of the two phases. The form of the quantities $\ldots \ldots$ appearing as the arguments in equation 49 guarantee that the diffusive body force is invariant under superposed rigid body velocities, and under superposed rigid-body angular velocities of the whole mixture. For a mixture of two ideal fluids, equation 49 is assumed to reduce to:

$$
\Psi=\Psi\left(\Gamma-\Lambda, \mathbf{u}-\mathbf{v}, \rho_{1}, \rho_{2}\right)
$$

which if $\Psi$ is assumed a linear function of the arguments takes the form:

$$
\Psi=-\frac{(\mathbf{u}-\mathbf{v})}{\rho d_{1}}+\operatorname{curl} \frac{(\mathbf{u}-\mathbf{v})}{\rho d_{2}}
$$

where $d_{1}$ and $d_{2}$ are functions of $\rho_{1}$ and $\rho_{2}$. If the principle of material frame-indifference is to be applied for all orthogonal transformations, then the second term in equation 51 must be omitted.

Green and Naghdi [1965] propose a dynamical theory of interacting continua and, as an example, study the mixture of two Newtonian compressible viscous fluids. They assume that if the diffusive force is a linear function of the velocity and the velocity gradients of each fluid, it can be represented by:

$$
f_{i}=a_{i}+B_{1}\left(u_{i}-v_{i}\right)+B_{2} e_{i j k}\left(\Gamma_{j k}-\Lambda_{j k}\right)
$$

where $a_{i}, B_{1}$, and $B_{2}$ are functions of $\rho_{1}, \rho_{2}$, and temperature $T$, and:

$$
\begin{aligned}
& \Gamma_{i k}=\frac{1}{2}\left[u_{i, k}-u_{k, i}\right] \\
& \Lambda_{i k}=\frac{1}{2}\left[v_{i, k}-v_{k, i}\right]
\end{aligned}
$$

where $u_{i}$ and $v_{i}$ are the velocities of the two fluids. Based on the standard thermody. namical arguments used in continuum mechanics (cf Coleman and Noll [1963]), Green and Naghdi [1965] show that $B_{1} \geq 0, B_{2} \geq 0$, and:

$$
a_{i}=\rho_{1} \frac{\partial A}{\partial \vartheta_{2}} \frac{\partial \vartheta_{2}}{\partial x_{i}}-\rho_{2} \frac{\partial A}{\partial \vartheta_{1}} \frac{\partial \vartheta_{1}}{\partial x_{i}}
$$


where $\vartheta_{1}=\frac{1}{\rho_{1}}, \vartheta_{2}=\frac{1}{\rho_{2}}$, and $A$ is the Helmholtz free energy function for the mixture.

Mills [1966] studies the mixture of Newtonian fluids and derives the incompressibility condition (or volume additivity), which is an additional constraint relating the densities of the two fluids in the reference configuration to that of the present configuration. This constraint is shown to be:

$$
\frac{\rho_{1}}{\rho_{10}}+\frac{\rho_{2}}{\rho_{20}}=1
$$

where $\rho_{10}$ and $\rho_{20}$ are the densities of each fluid per unit volume of that fluid and $\rho_{1}$ and $\rho_{2}$ are the densities of each fluid per unit volume of the mixture. Mills [1966] also assumes that the diffusive force is given by:

$$
f_{i}=a_{i}+B_{1}\left(u_{i}-v_{i}\right)+B_{2} e_{i j k}\left(\Gamma_{j k}-\Lambda_{j k}\right),
$$

and he shows that for an incompressible mixture of two Newtonian fluids, it takes the form:

$$
\begin{aligned}
f_{i} & =-\left[\frac{p}{\rho_{10}} \frac{\partial \rho_{1}}{\partial x_{i}}+\frac{\partial A}{\partial \rho}\left(\rho_{2} \frac{\partial \rho_{1}}{\partial x_{i}}-\rho_{1} \frac{\partial \rho_{2}}{\partial x_{i}}\right)\right] \\
& +B_{1}\left(u_{i}-v_{i}\right)+B_{2} e_{i j k}\left(\Gamma_{j k}-\Lambda_{j k}\right)
\end{aligned}
$$

where $p$ is an arbitrary scalar, $A$ is the Helmholtz energy function for the mixture, and $B_{1}$ and $B_{2}$ are assumed to be functions of $\rho$ and $T$.

Crochet and Naghdi [1966] study the flow of fluid through an elastic solid, using mixture theory. They propose the following form for the diffusive force:

$$
f=f_{0}+f_{e}
$$

where $\mathfrak{f}_{0}$ is the equilibrium value of $\mathbf{f}$ (when the velocity gradients and the relative velocity all vanish). If the solid is an elastic material, Crochet and Naghdi [1966] show that:

$$
\mathbf{f}_{\mathrm{o}}=\rho_{1} \frac{\partial A}{\partial \rho_{2}} \operatorname{grad} \rho_{2}-\rho_{2} \operatorname{tr}\left[A_{F}^{(1)} \operatorname{grad~F}{ }^{(1)}\right]
$$

where $A$ is again the Helmholtz free energy function, and $F^{(1)}$ is the displacement gradient for the solid phase, i.e.:

$$
\mathbf{F}^{(1)}=\operatorname{grad} \mathbf{x}(\mathbf{X}, t)
$$

and in the above expression:

$$
\operatorname{tr}\left[A_{F}^{(1)} \operatorname{grad} \mathbf{F}^{(1)}\right]=\frac{\partial A}{\partial x_{j, k}} x_{j, k l} X_{l, i}
$$

Green and Naghdi [1967] develop a theory of mixtures where the energy equation and the entropy production inequality are postulated for each constituent. For a mixture of two ideal fluids they obtain:

$$
f_{i}=\frac{\rho_{1}}{\rho}\left[\rho_{1} \frac{\partial A_{1}}{\partial \vartheta_{2}}+\rho_{2} \frac{\partial A_{2}}{\partial \vartheta_{2}}\right] \frac{\partial \vartheta_{2}}{\partial x_{i}}-\frac{\rho_{2}}{\rho}\left[\rho_{1} \frac{\partial A_{1}}{\partial \vartheta_{1}}+\rho_{2} \frac{\partial A_{2}}{\partial \vartheta_{1}}\right] \frac{\partial \vartheta_{1}}{\partial x_{i}}
$$




$$
+\left(D_{1}-D_{2}\right)\left(v_{i}^{(1)}-v_{i}^{(2)}\right)
$$

where $\vartheta_{1}=\frac{1}{p_{1}}, \vartheta_{2}=\frac{1}{p_{2}}$, and:

$$
\begin{aligned}
& A_{1}=A_{1}\left(\vartheta_{1}, \vartheta_{2}, \theta_{1}, \theta_{2}\right) \\
& A_{2}=A_{2}\left(\vartheta_{1}, \vartheta_{2}, \theta_{1}, \theta_{2}\right)
\end{aligned}
$$

Müller [1968] develops a thermodynamic theory for mixtures of fluids, using mixture theory and invoking principles of frame-indifference, equipresence, etc. He assumes that the interactive force is a function of:

$$
f_{i}=f_{i}\left(\rho_{\alpha}, \frac{\partial \rho_{\alpha}}{\partial x_{j}}, v_{i}^{\alpha}, \frac{\partial v_{i}^{\alpha}}{\partial x_{j}}, \theta, \frac{\partial \theta}{\partial x_{j}}\right), \quad \alpha=1,2
$$

where $\theta$ is the tempe ature of the mixture. Applying the principle of material frame indifference, he shows that equation 66 ; for a binary mixture, should take the form:

$$
f_{i}=f_{i}\left(\rho_{\alpha}, \frac{\partial \rho_{\alpha}}{\partial x_{j}}, V_{i}^{12}, D_{i j}^{\alpha}, \Omega_{i j}^{12}, \theta, \frac{\partial \theta}{\partial x_{j}}\right)
$$

where:

$$
\begin{gathered}
V_{i}^{12}=v_{i}^{1}-v_{i}^{2} \\
D_{i j}^{\alpha}=\frac{1}{2}\left(\frac{\partial v_{i}^{\alpha}}{\partial x_{j}}+\frac{\partial v_{j}^{\alpha}}{\partial x_{i}}\right) \quad \alpha=1,2 \\
\Omega_{i j}^{12}=\frac{1}{2}\left(\frac{\partial v_{i}^{1}}{\partial x_{j}}-\frac{\partial v_{j}^{1}}{\partial x_{i}}\right)-\frac{1}{2}\left(\frac{\partial v_{i}^{2}}{\partial x_{j}}-\frac{\partial v_{j}^{2}}{\partial x_{i}}\right)
\end{gathered}
$$

Müller [1968] then shows that a further consequence of the principle of material frameindifference is that the constitutive function $f_{i}$ must be an isotropic vector function relative to the orthogonal group. Using representation theorems ( c.f. Truesdell and Noll (1965]), and assuming that $f_{i}$ is linear in the variables, Müller [1968] proposes the following relation for $f_{i}$ :

$$
f_{i}=f_{1} \frac{\partial \theta}{\partial x_{i}}+\sum_{\beta=1}^{2} f_{\rho \beta} \frac{\partial \rho_{\beta}}{\partial x_{i}}+f_{2} V_{i}^{12}
$$

where the coefficients $f_{1}, f_{\rho \beta}$, and $f_{2}$ can depend on $\rho_{\beta}$ and $\theta$,

In a later paper, Green and Naghdi [1968] generalize their earlier work so as to include the temperature gradient and the density gradients in their formulation. Thus, they show that:

$$
\begin{gathered}
f_{k}=a_{k}+a \frac{\partial \theta}{\partial x_{k}}+D\left(u_{k}-v_{k}\right) \\
a_{k}=\rho_{1} \frac{\partial F}{\partial \rho_{2}} \frac{\partial \rho_{2}}{\partial x_{k}}-\rho_{2} \frac{\partial F}{\partial \rho_{1}} \frac{\partial \rho_{1}}{\partial x_{k}}+\frac{\partial \phi}{\partial x_{k}}
\end{gathered}
$$


where $\phi=\phi\left(\rho_{1}, \rho_{2}, \theta\right)$ and $F$ is the Helmholtz free energy at equilibrium, i.e., $A=$ $F\left(\rho_{1}, \rho_{2}, \theta\right)$.

Craine [1971] studies the steady oscillation of an infinite plate in a binary mixture of incompressible Newtonian fluids. The form that he uses for the interactive force is:

$$
f_{i}=\alpha\left(v_{i}^{(1)}-v_{i}^{(2)}\right)+\beta \frac{\partial \rho}{\partial x_{i}}+\gamma \frac{\partial \theta}{\partial x_{i}}
$$

where $\rho=\rho_{1}+\rho_{2}$ and $\alpha, \beta$, and $\gamma$ are functions of $\rho$ and $\theta$. From theimodynamic restrictions due to the Clausius.Duhem inequality Craine [1971] shows that:

$$
\beta=-\lambda
$$

where $\lambda$ is a Lagrange multiplier associated with the constraint of incompressibility of the mixture, i.e.:

$$
\left(\rho_{20}-\rho\right) \operatorname{tr}^{1} \mathbf{D}+\left(\rho-\rho_{10}\right) \operatorname{tr}^{2} \mathbf{D}-(\operatorname{grad} \rho) \cdot\left(\mathbf{u}^{(1)}-\mathbf{u}^{(2)}\right)=0
$$

where:

$$
\begin{gathered}
\rho_{10}=\frac{\rho_{1}}{\gamma} \\
\rho_{20}=\frac{\rho_{2}}{(1-\gamma)}
\end{gathered}
$$

are the reference densities of the two constituents, when separated. It should be noted that equations 77 and 78 imply that:

$$
\frac{\rho_{1}}{\rho_{10}}+\frac{\rho_{2}}{\rho_{20}}=1
$$

Drew [1976] studies the effect of lift and Brownian motion. He writes the interphase force, $f_{I}$, as:

$$
\mathrm{f}_{I}=\mathrm{f}_{\mathrm{p}}+\mathrm{f}
$$

where $f_{p}$ is the pressure force on the particles and $f$ represents the effect of other forces such as drag lift, etc., on the particles. Following Drew and Segel [1971a], $f_{p}$ has the form:

$$
f_{p}=p_{f} g r a d \nu
$$

where $p_{f}$ is the fluid pressure and $\nu$ is the volume fraction of the solid phase.

In addition to using the "principle of phase separation"10 (Drew and Segel, 1971a) ("a bulk-phase variable should depend only on variables from that same phase"), Drew [1976] also introduces two principles:

\footnotetext{
10This so-called principle that is often attributed to Drew and Segel [1871a] is actually first put forward as an assumption by Adkins [1963a]:

It is assumed that within the mixture, the properties of any given component are defined by means of constitutive equations relating the partial stress tensor, the density (or concentration) and the kinematic quartities, such as velocity gradients, acceleration gradients and their successive time dorivatives, for that component.
}

Furthermore, Adkins [1963a] points out that: 
1. The principle of local dependence on dispersed phase variables states that nonlocal effects (specifically gradients) of dispersed phase variables should be omitted from constitutive relations.

2. The principle of correct low concentration limits states that when the dispersed phase is sufficiently dilute, the mixture behaves as if it were made up of the continuous phase alone. ${ }^{11}$

Based on these three "principles," which perhaps should be called "assumptions," Drew proposes a general isotropic linear dependence of $f_{I}$ on $\mathbf{u}-\mathbf{v}, \mathrm{D}_{f}$, and $\operatorname{grad}(\operatorname{div} \mathbf{v})$ given by:

$$
\mathbf{f}_{I}=\alpha S(\mathbf{u}-\mathbf{v})+\alpha B \Delta \mathbf{v}+C \operatorname{grad}(\operatorname{div} \mathbf{v})+L \mathrm{D}_{f}(\mathbf{u}-\mathbf{v})
$$

where $\mathbf{u}$ is the velocity of the solid (dispersed) phase; $\mathbf{v}$ is the velocity of the fluid (continuous) phase; $D_{f}$ is the rate of deformation tensor for the fluid; $\Delta$ is the Laplacian operator; and the quantities $S, L, B$, and $C$ are scalar functions of the invariants, which can be constructed from $\mathbf{u}-\mathbf{v}, \mathbf{D}_{f}$, and $\Delta \mathbf{v}$. It should be noted that these "principles," especially the "principle of local dependence," put severe restrictions on the structure of the dispersed (solid) stress tensor. This subject matter is discussed in Massoudi and Boyle [1987] .

The first term in equation 82 is the drag term, the second and third terms are called viscous forces (which do not involve the particle motion), and the fourth term is the lift force. The drag force, in general, has the form:

$$
\mathbf{f}_{D}=\nu S\left(\nu, \mu_{f}, \rho_{\mathbf{s}}, \rho_{f},|\mathbf{u}-\mathbf{v}|, I_{\mathrm{D}}, I I_{\mathbf{D}}, I I I_{\mathrm{D}}\right)(\mathbf{u}-\mathbf{v})
$$

where $I_{\mathrm{D}}, I I_{\mathrm{D}}$, and $I I I_{\mathrm{D}}$ are the principle invariants of $\mathrm{D}_{f}$, and the lift force has the form:

$$
\mathbf{f}_{L}=L\left(\nu, \mu_{f}, \rho_{\mathbf{s}}, \rho_{f},|\mathbf{u}-\mathbf{v}|, I_{\mathbf{D}}, I I_{\mathbf{D}}, I I I_{\mathbf{D}}\right) \mathbf{D}_{f}(\mathbf{u}-\mathbf{v})
$$

Using the principle of low concentration limit, Drew [1976] shows that as $\nu \rightarrow 0$ :

$$
\begin{gathered}
S \rightarrow \frac{9 \mu_{f}}{2 a^{2}}(1+6.55 \nu), \\
C \rightarrow 0,
\end{gathered}
$$

It would be possible, for example, to postulate that the partial stress tensor for a given fluid should be dependent not only upon the kinematical quantities defined for the fluid itself $\hat{f}$ but also upon the concentration, velocity gradients, acceleration gradients, and time derivatives of these quantities defined, at the point under consideration, for the other fluids in the mixture. This would give an explicit coupling of mechanical properties.

Later, Green and Adkins [1964] use this generalization and formulate a general theory of non-linear diffusion where they study a mixture of two non-Newtonian fluids, and the problem of the diffusion of a non-Newtonian fluid through a Cauchy elastic solid.

${ }^{11}$ Interestingly, a similar idea is put forward earlier by Green and Adkins [Green and Adkins, 1964]. In their formulation for a mixture of non-Newtonian fluids, they state: "Also, in the absence of one fluid the constitutive equation for the other must reduce to the usual one for that fluid alone." 


$$
\begin{gathered}
B \rightarrow \frac{3}{4} \mu_{f} \\
L \rightarrow \frac{-3(6.46) \nu}{8 \pi}\left(\rho_{f} \mu_{f}\right)^{\frac{1}{2}}\left|\mathbf{D}_{f}\right|^{-\frac{1}{2}},
\end{gathered}
$$

where $\nu$ is the volume fraction of solid. The effect of Brownian motion on the particles is included in the force $-K_{a} \operatorname{grad} \nu$.

In a later paper, Drew et al. [1979] considers the phase separation mechanism and its relationship with the effect of virtual mass during the acceleration of a two-phase mixture. An important contribution of this work, though largely performed previously by El-Kaissy [1975], is the derivation of objective virtual mass accelerations. Drew et al. claim that the most general objective acceleration $a_{v m}$ for two-phase flow has the structure:

$$
\begin{gathered}
\mathbf{a}_{v m}=\left[\frac{D^{s} \mathbf{u}}{D t}-(\operatorname{grad} \mathbf{u})(\mathbf{u}-\mathbf{v})\right]-\left[\frac{D^{f} \mathbf{v}}{D t}-(\operatorname{grad} \mathbf{v})(\mathbf{v}-\mathbf{u})\right] \\
+(1-\lambda)[\operatorname{grad}(\mathbf{v}-\mathbf{u})](\mathbf{u}-\mathbf{v})
\end{gathered}
$$

where

$$
\frac{D^{\prime}}{D t}=\frac{\partial}{\partial t}+[\operatorname{grad}(\cdot)] \mathbf{u}
$$

and

$$
\frac{D^{f}}{D t}=\frac{\partial}{\partial t}+[\operatorname{grad}(\cdot)] \mathbf{v}
$$

where $\lambda$ is a parameter, superscripts $s$ and $f$ refer to the solid and fluid phases, respectively, and as before $\mathbf{u}$ is the velocity of the solid phase and $\mathbf{v}$ the fluid phase. Equation 89 can be written in several forms. Two examples are given by Drew et al. [1979]:

$$
\mathbf{a}_{v m}=\frac{D^{f} \mathbf{u}}{D t}-\frac{D^{\mathbf{a}} \mathbf{v}}{D t}+(1-\lambda)[\operatorname{grad}(\mathbf{v}-\mathbf{u})](\mathbf{u}-\mathbf{v})
$$

or

$$
\mathbf{a}_{v m}=\frac{D^{\bullet}(\mathbf{u}-\mathbf{v})}{D t}+[(\lambda-2) \operatorname{grad} \mathbf{u}+(1-\lambda) \operatorname{grad} \mathbf{v}](\mathbf{u}-\mathbf{v}),
$$

Wallis [1969], Hinze [1975], and Soo [1967] use $\frac{D^{*}(\mathbf{u}-\mathbf{v})}{D^{t}}$ as the virtual mass acceleration term in their investigations. Equation 93 clearly indicates that this tern, alone, is not frame-indifferent (see Appendix A) and is, thus, not a valid choice for $\mathrm{a}_{v m}$.

Shi, Rajagopal, and Wineman [1981\} use mixture theory to study the diffusion of an ideal fluid through a non-linear elastic media. They assumed that all the constitutive functions depend upon:

$$
\Lambda=\Lambda\left(\mathbf{F}, \rho_{2}, \nabla \mathbf{F}, \operatorname{grad} \rho_{2}, T, \operatorname{grad} T, \mathbf{u}, \mathbf{v}, \mathbf{L}, \mathbf{M}\right)
$$


where $\mathbf{F}$ is the deformation gradient of the solid continua, $\rho_{2}$ the density of fluid measured per unit volume of the mixture, $T$ the ternperature of mixture, $u$ and $v$ are the velocities of solid and fluid, respectively, and $L$ and $M$ are velocity gradients of the fluid and solid phases, respectively. Following Crochet and Naghdi [1966], Shi e.t al. [1981] also assume that the diffusive force can be written as the sum of a static part and a dynamic part, i.e.:

$$
\overline{\mathbf{f}}=\overline{\mathbf{f}}^{d}+\overline{\mathbf{f}}^{d}
$$

Based on the balance of energy and the application of the Clausius-Duhem inequality, they show that:

$$
\overline{\mathrm{f}}_{k}^{s}=-\rho_{2} \frac{\partial F_{i j}}{\partial x_{k}} \frac{\partial A}{\partial F_{i j}}+\rho_{1} \frac{\partial A}{\partial \rho_{2}} \frac{\partial \rho_{2}}{\partial x_{k}}-\frac{p}{\rho_{10}} \frac{\partial \rho_{1}}{\partial x_{k}}
$$

where $A=A\left(F_{i j}, p, T\right)$, and $p$ is an indeterminate scalar arising from the use the constraint of incompressibility of the mixture, i.e.:

$$
\frac{\rho_{1}}{\rho_{10}}+\frac{\rho_{2}}{\rho_{20}}=1
$$

Shi et al. [1981] assume the following form for the dynamic part of the interactive force:

$$
\overline{\mathbf{f}}_{k}^{d}=c_{2}\left(u_{k}-v_{k}\right)
$$

where $c_{2}$ is a function of $\rho_{1}, \rho_{2}$ and $T$, and the entropy inequality indicates that $c_{2} \geq 0$. Therefore, the general form for 'be interactive force, used by Shi et al. [1981] is given as:

$$
\overline{\mathbf{f}}_{k}=\frac{\partial \phi_{1}}{\partial x_{k}}+\overline{\mathbf{f}}_{k}^{s}+\overline{\mathbf{f}}_{k}^{d}
$$

where:

$$
\phi_{1}=\rho_{1}\left(\dot{A_{1}}-A\right)
$$

and $A_{1}$ is the Helmholtz free energy of the solid continua.

Shi et al. [1981] assume that the solid phase was initially isotropic, hence, the dependence on $F_{i j}$ is replaced by $C_{i j}=F_{k i} F_{k j}$ and the free energy function $A$ is expressed as:

$$
A=A\left(I_{1}, I_{2}, I_{3}, \rho_{2}, T\right)
$$

where:

$$
\begin{gathered}
I_{1}=C_{i i} \\
I_{2}=\frac{1}{2}\left(I_{1}^{2}-C_{i k} C_{i k}\right) \\
I_{3}=\operatorname{det}\left|C_{i j}\right|
\end{gathered}
$$

The above relations, the fact that $\rho_{1} \operatorname{det} \mathbf{F}=\rho_{10}$ and the constraint of incompressibility, given by equation 97 , imply:

$$
I_{3}^{-\left(\frac{1}{2}\right)}=1-\frac{\rho_{2}}{\rho_{20}}
$$


the expression for equation 106 is given as:

$$
\begin{gathered}
\mathbf{f}_{k}=\frac{\partial \phi_{1}}{\partial x_{k}}-\frac{p}{\rho_{10}} \frac{\partial \rho_{1}}{\partial x_{k}}+\rho_{1} \frac{\partial A}{\partial \rho_{2}} \frac{\partial \rho_{2}}{\partial x_{k}}+\alpha \frac{\rho_{1}}{\rho_{10}} \frac{\rho_{2}}{\rho_{20}}\left(u_{k}-v\right) \\
-\rho_{2}\left[\left(\frac{\partial A}{\partial I_{1}}+I_{1} \frac{\partial A}{\partial I_{2}}\right) \delta_{i j}-\frac{\partial A}{\partial I_{2}} C_{i j}\right] \frac{\partial C_{i j}}{\partial x_{k}}
\end{gathered}
$$

Now, to evaluate $f_{k}$, an expression for the free energy function is needed. The approach given by Shi, Rajagopal, and Wineman [1981] is a very general approach and in principle can be applied to any multicomponent system, including fluid-solid flows. In their study, they are interested in the diffusion of a fluid through rubber, where its free energy function for use in swelling is given by Treloar [1975] as:

$$
\begin{gathered}
A=K\left(I_{1}-3-\frac{1}{2} \ln I_{3}\right) \\
K=\frac{1}{2} \frac{R T}{M_{c}}
\end{gathered}
$$

where $R$ is the gas constant, $T$ is absolute temperature and $M_{c}$ is the molecular weight between cross-links. Therefore, using equation 105 in equation 107 and applying the results to equation 106, Shi et al. shows that:

$$
f_{k}=\frac{\partial \phi_{1}}{\partial x_{k}}-\frac{p}{\rho_{10}} \frac{\partial \rho_{1}}{\partial x_{k}}-K \frac{\rho_{10}}{\rho_{20}} \frac{\partial \rho_{2}}{\partial x_{k}}-\rho_{2} K \frac{\partial I_{1}}{\partial x_{k}}+C_{2}\left(u_{k}-v\right)
$$

Nunziato [1983] develops a theory for fluid-particle flows based on mixture theory. He defines the extra momentum transfer $\mathbf{n}_{a}$ as:

$$
\mathbf{n}_{\mathrm{a}}=\mathbf{f}_{\mathrm{a}}-\pi \nabla \phi_{\mathrm{a}}
$$

where $\phi_{a}$ is the volume fraction of the a-th constituent, and $\pi$ is a Lagrange multiplier associated with the incompressibility of the mixture. Nunziato [1983] also defines the extra stress tensor $\tau_{a}$ as:

$$
\tau_{a}=\sigma_{a}+\phi_{a} p_{a} I
$$

where $p_{a}$ is a Lagrange multiplier. Because of these definitions, in the linear mornentum equation for constituent $a$, Nunziato obtains the following:

$$
\left(\pi-p_{a}\right) \nabla \phi_{a}
$$

which he calls the "diffusive force", which "can account for the contact pressure at high concentrations (cf. Anderson and Jackson [1967]) and the diffusive pressure associated with the Brownian motion of small particles at dilute concentrations (cf. Drew [1976])." He proposes the following constitutive relations:

$$
\mathbf{n}_{f}=-\phi_{a} \mathbf{F}\left(\mathbf{u}_{f}-\mathbf{u}_{a}\right)=-\mathbf{n}_{a}^{+}
$$


where $\mathbf{F}$ is defined as the "drag tensor," which is a linear isotropic function of $\mathbf{D}_{f}$ :

$$
\mathbf{F}=\alpha_{1} \mathbf{I}+2 \alpha_{2} \mathbf{D}_{f}
$$

where $\alpha_{1}$ and $\alpha_{2}$ may be scalar valued functions of $\phi_{a}$ and the invariants of $D_{f}$. Nunziato indicates that the drag forces are characterized by the diagonal of $F$, while ( $F-\operatorname{diag} F$ ) indicates the lift forces. As $\phi_{1} \rightarrow 0, \alpha_{1}$ and $\alpha_{2}$ must reduce to their appropriate low concentration limits (cf, Butchelor [1972] and Saffman [1965], respectively), which are:

$$
\begin{gathered}
\alpha_{1} \rightarrow \frac{9}{2} \frac{\mu_{f}}{a^{2}}\left(1+5.55 \phi_{s}\right) \\
\alpha_{2} \rightarrow \frac{3(3.23)}{4 \pi a}\left(\frac{\rho_{f} \mu_{f}}{\left|D_{f}\right|}\right)^{\frac{1}{2}}
\end{gathered}
$$

where $a$ is the particle radius. Furthermore, he suggests the following relations for the pressures:

$$
\begin{gathered}
\pi-p_{f}=\xi_{f} \dot{\phi}_{f}+\omega_{f}\left(\mathbf{u}_{f}-\mathbf{u}_{s}\right) \cdot\left(\mathbf{u}_{f}-\mathbf{u}_{s}\right) \\
\pi-p_{s}=-\beta_{s}+\xi_{s} \dot{\phi}_{s}
\end{gathered}
$$

where:

$$
\beta_{\Delta}=\rho_{a} \frac{\partial A_{0}}{\partial \phi_{a}}
$$

where $A_{B}$ is the free energy of the solid particles, $\xi_{f}$ and $\xi_{a}$ were called bulk viscosities, and $\pi$ can be thought of as interface pressure, and:

$$
\grave{\phi}_{a}=-\phi_{a} \operatorname{div} \mathbf{u}_{a}
$$

Passman et al. [1983] develops a theory of multiphase mixtures and proposes the following constitutive relationship for $\pi$ :

$$
\pi=p_{a}-\beta_{a}+\phi_{a} \lambda_{a} \operatorname{div} u_{a}+\operatorname{div}\left(A_{a} \operatorname{grad} \phi_{a}\right), \quad a=1,2
$$

where $\beta_{a}$ is the configuration pressure. Writing this equation for both constituents and eliminating $\pi$, they show that;

$$
p_{1}-p_{2}=\beta_{1}-\beta_{2}+\operatorname{div}\left[\left(A_{1}+A_{2}\right) \operatorname{grad} \phi_{1}\right]+\phi_{1} \lambda_{1} \operatorname{div} \mathbf{u}_{1}+\left(1-\phi_{1}\right) \lambda_{2} \operatorname{div} \mathbf{u}_{2}
$$

where $\beta_{a}, A_{a}$, and $\lambda_{a}$ are functions of the volume fraction $\phi_{a}$. They assume $\beta_{2}=0$ (fluid), and give the following explanation: "Physically, the expression 122 asserts that the differences in the pressures of the solid and the fluid result from three effects: intergranular contact forces that are represented by $\beta_{1}$, local variations in the stress field due to a non-homogeneous particle disiribution that are represented by the moduli $A_{1}$, $A_{2}$, and bulk viscous forces due to intergranular fiction and the fluid viscosity which are represented by the viscosities $\lambda_{1}, \lambda_{2}$." Based on the work of Stuhmiller [1977], they suggest:

$$
\pi=p_{a}-\beta_{a}+\lambda_{a} \phi_{a} \operatorname{div} \mathbf{u}_{a}+\omega_{a}\left(\mathbf{u}_{a}-\mathbf{u}_{b}\right) \cdot\left(\mathbf{u}_{a}-\mathbf{u}_{b}\right) \quad(a \neq b)
$$


which by eliminating $\pi$ between the two phases becomes:

$$
p_{1}-p_{2}=\beta_{1}-\beta_{2}+\phi_{1} \lambda_{1} \operatorname{div} \mathbf{u}_{1}-\left(1-\phi_{1}\right) \lambda_{2} \operatorname{div} \mathbf{u}_{2}+\left(\omega_{1}-\omega_{2}\right)\left(\mathbf{u}_{1}-\mathbf{u}_{2}\right) \bullet\left(\mathbf{u}_{1}-\mathbf{u}_{2}\right)
$$

In their study of particle segregation in Poiseuille flow, Nunziato and Mc'Tigue [1984] assumed that:

$$
f_{f}=-\phi_{0} \mathbf{F}\left(u_{f}-u_{a}\right)+\phi_{s} \eta \operatorname{div} D_{f}-p_{f} \operatorname{grad} \phi_{a}
$$

where $\mathrm{F}$ is given by equation 114 . They also state that the second term on the righthand side of equation 125 represents the Faxen forces arising in shear flows where in the low concentration limit:

$$
\eta \rightarrow \frac{3}{4} \mu_{f}
$$

In contrast to previous studies based on mixture theory, where the force on one phase was equal and opposite to the force on the other phase (summation laws in mixture theory ), Nunziato and McTigue (1984) propose:

$$
\sum \mathfrak{f}_{\mathfrak{a}}=\operatorname{div} \mathbf{S}
$$

where $\mathrm{S}$ is called the interaction stress ( cf. Williams [1978]). Therefore:

$$
\mathbf{f}_{\mathbf{a}}=\operatorname{div} \mathbf{S}-\mathbf{f}_{f}
$$

where $f_{a}$ is the momentum transfer for the solid particles. Based on the work of Lin et al. [1970], Nunziato and McTigue assume:

$$
\mathrm{S}=2 \phi_{s} \mu_{\diamond} \mathrm{D}
$$

where in the case of particles moving with the fluid, the mixture stress $T$ defined as:

$$
\mathbf{T}=\sum\left(\mathbf{T}_{a}-\rho_{a} \mathbf{u}_{a} \otimes \mathbf{u}_{a}\right)+\rho \mathbf{u} \otimes \mathbf{u}+\mathbf{S}
$$

results in the correct effective viscosity to within $\left.\alpha \phi_{s}^{2}\right)$ if:

$$
\mu_{s}=\frac{7}{2} \mu_{f}
$$

This is based on Einstein's analysis. Note that $\mathrm{u}$ in equation 130 is the velocity of the mixture defined by:

$$
\rho \mathbf{u}=\rho_{1} \mathbf{u}_{\mathfrak{s}}+\rho_{2} \mathbf{u}_{f}
$$

Later, Passman et al. (1986) studies the shearing motion of a fluid-saturated granular material using mixture theory. They assume that:

$$
\mathbf{f}_{1}=-\mathbf{f}_{2}=\mathbf{f}_{1}^{e}\left(\theta, \phi_{1}, \operatorname{grad} \phi_{1}\right)-D\left(\theta, \phi_{1}\right)\left(\mathbf{u}_{1}-\mathbf{u}_{2}\right)
$$

where $D$ is the drag coefficient such that $D \geq 0$. They show that:

$$
\mathbf{f}_{a}^{e}=-\pi \operatorname{grad} \phi_{a}
$$


where $\pi$ is again the interface pressure, for which they also propose a constitutive equation. In their numerical study of simple shearing flow, Passman et al. [1986] assume that $D$ is constant.

In their formulation of continuum theories for suspensions, Nunziato et al. [1986] propose the following constitutive relations:

$$
f_{f}+f_{b}=\operatorname{div} S
$$

where:

$$
\mathbf{f}_{\theta}=p_{f} \operatorname{grad} \phi_{b}+\phi_{b} \mathbf{F}_{1}\left(\mathbf{u}_{f}-\mathbf{u}_{a}\right)+2 \phi_{6} \mathbf{F}_{2} \operatorname{div} \mathrm{D}_{f}
$$

where:

$$
\mathbf{F}_{i}=\alpha_{j} \mathbf{I}+2 \beta_{i} \mathbf{D}_{f}, \quad(i=1,2)
$$

They identify the first term in equation 136 as the net buoyant force on the particles, while $\alpha_{1}$ and $\alpha_{2}$ represent Stokes drag and the Faxen force on the particles, respectively. The coefficient $\beta_{1}$ corresponds to a lift force given by Saffman [1965] and $\beta_{2}$ corresponds to a lift (or lateral) force identified by Ho and Leal [1974] (See Appendix B.). Based on Einstein's [1956] result that the mixture viscosity increases with the volume fraction, Nunziato et al. [1986] assume:

$$
\mathrm{S}=\phi_{\theta} \eta \mathrm{D}_{f}
$$

It should be noted that this equation does not agree with equation 129 proposed earlier by Nunziato and Mc'Tigue [1984] .

McTigue et al. [1986] study the rheological effects of nonuniform particle distribution in dilute suspensions. Their formulation of the theory is basically that of Nunziato et al. [1986] given by equations $135-137$. They provide the following low concentration forms for the coefficients $\alpha_{i}$ and $\beta_{i}$ :

$$
\begin{gathered}
\alpha_{1} \rightarrow \frac{9 \mu_{f}}{2 a^{2}} \\
\alpha_{2} \rightarrow \frac{3(6.46)}{4 \pi a}\left(\frac{\rho_{f}^{2} \mu_{f}^{2}}{2 \operatorname{tr} \mathrm{D}_{f}^{2}}\right)^{\frac{1}{4}}, \\
\beta_{1} \rightarrow \frac{7}{4} \mu_{f}, \\
\beta_{2} \rightarrow-\left[\frac{\left(\operatorname{tr} \mathrm{D}_{f}^{2}\right)^{\frac{1}{2}}}{\left|\operatorname{div} \mathrm{D}_{f}\right|} g_{1}-g_{2}\right]
\end{gathered}
$$

where $g_{1}$ and $g_{2}$ are functions that depend on the geometry of the flow. They assume:

$$
\mathrm{S}=2 \phi_{\bullet} \eta \mathrm{D}
$$

where $\eta$ is defined as an interaction viscosity, and $\mathbf{D}$ is the deformation rate of the mixture. Agan, this definition, in general, is different from those given earlier by the same authors (cf. equations 129 and 138 ). 
Passman [1986] derives a general representation for the interactive force. He assumes that:

$$
f=f(w, d, D)
$$

where $w$ and $d$ are objective vectors and $D$ is a symmetric objective tensor (See Appendix A). Passman then shows that by using the representation theorem for isotropic functions, equation 144 can be written as:

$$
\begin{aligned}
f= & \alpha_{1}(\Lambda) \mathbf{w}+\alpha_{2}(\Lambda) \mathrm{Dw}+\alpha_{3}(\Lambda) \mathbf{D}^{\prime \prime} \mathrm{w} \\
& +\beta_{1}(\Lambda) \mathrm{d}+\beta_{2}(\Lambda) \mathrm{Dd}+\beta_{3}(\Lambda) \mathrm{D}^{2} \mathrm{~d}
\end{aligned}
$$

where:

$$
\begin{aligned}
& \Lambda=\Lambda\left[I_{1}, I_{2}, I_{3}, w \bullet w, w \bullet D w, w \bullet D^{2} w\right. \\
& \left.d \bullet d, d \bullet D d, d \bullet D^{2} d, w \bullet d, w \bullet D d, w \bullet D^{2} d\right]
\end{aligned}
$$

and $I_{1}, X_{2}$, and $I_{3}$ are the principal invariants of $D$.

\subsection{Generalization of Single Particle Results}

\subsubsection{Drag}

The relationship between the drag force and velocity of a single particle is well established [Khan and Richardson, 1987]. Unfortunately, the large quantity of experimental data and variety of correlations available for the single particle do not exist for mixtures containing large numbers of particles. An exception to this is the special case of fluidized beds. Due to the relative ease of performing experiments in fluidized beds, and the simple force balance involved, a large amount of data is collected and correlated. Since the data is correlated using the Galileo number (a dimensionless buoyancy force), it is not easily applied to more general flow situations. Barnea and Mizrahi [1973] give a critical review of publications, up to 1973, concerning the drag coeffcient for an assembly of spherical particles. A more recent extensive review of the various drag correlations for fluidized beds is given by Khan and Richardson [1989].

Several authors obtain drag force expressions for application to mixtures, typically using one of two approaches. They either propose modifications to the available drag correlations for single particles, or they apply a correction to the Stokes formula for drag on a single sphere. Note that we are considering only laminar flow in which the solid, or dispersed, phase is made up of spheres. Ishii and Zuber [1979] and Ishii and Chawla [1979] discuss drag correlations for mixtures in other flow regimes.

To use existing single particle drag correlations requires the assumption that complete similarity exists for the functional form of the drag coefficient versus Reynolds number curve. The advantage of this approach is that the extensive data for the single particle case can be used to find the drag force (at least approximately) between 
the phases of the mixture. Perhaps the simplest approximation that has been ased is |Rudinger, 1960):

$$
f_{m}=\nu \frac{f_{d}}{V_{p}^{\prime}}=\nu^{\frac{1}{2} \rho \pi a^{2} C_{D} \nu_{r}^{2}} \frac{\frac{1}{3} \pi a^{3}}{2}
$$

or simplified and writien in vector form:

$$
\mathrm{f}_{m}=\frac{3 \rho \underline{\rho}}{8} \frac{\underline{u}}{a} C_{D}\left|\mathrm{v}_{\mathrm{r}}\right| \mathrm{v}_{\mathrm{r}}
$$

where $f_{m}$ is the multiple particle (mixture) drag force per unit volume, $f_{o}$ is the singlo particle drag force, $\nu$ is the volume fraction of solid, $\rho$ is the density of the fluid, $a$ is the radius of the spheres, $C_{D}$ is the single particle drag coefficient, and $v_{r}$ is the relative velocity between phases. Ishii and Zuber (1979) use a single particle correlation with a modified (mixture) Reynolds number. The mixture Reynolds number is based on a mixture viscosity defined as:

$$
\frac{\mu_{m}}{\mu_{f}}=\left(1-\frac{\nu}{\nu_{m}}\right)^{-2.6 \nu_{m} \mu^{*}}
$$

where $\mu_{m}$ is the mixture viscosity, $\mu_{f}$ is the fluid viscosity, $\nu$ is the volume fraction of the solid phase, $\nu_{m}$ is maximum packing fraction, and $\mu^{*}$ is defined by:

$$
\mu^{*}=\frac{\mu_{0}+0.4 \mu_{f}}{\mu_{a}+\mu_{f}}
$$

where $\mu_{\text {a }}$ is the viscosity of the solid phase. This correlation is intended for a number of different flow situations; for the special case of solid particles in a fluid equation 149 may be approximated as:

$$
\mu_{m} \approx \mu_{f}(1-1.6 \nu)^{-4}
$$

Drag force is then calculated using a particle Reynolds number defined by:

$$
R e=\frac{2 a \rho, v_{r}}{\mu_{m}}
$$

to get $C_{D}$ from a single particle correlation, and using $C_{D}$ in the relation:

$$
f_{D}=\frac{1}{2} \rho_{f} \pi a^{2} C_{D} v_{r}^{2}
$$

The derivation of corrections for Stokes drag formula has a disadvantage over the methods discussed above in that the results are strictly valid only for low Reynolds number (i.e., less than one). An advantage of working with low Re expressions is that more rigorous theoretical calculations are possible.

Drew [1976] obtains an approximate expression for drag through a simple manipulation of Batchelor's [1972) result for sedimentation of spheres. Batchelor considers the sedimentation of a dilute suspension of identical small rigid spheres and obtains the result that the mean sedimentation velocity (correct to order $\nu$ ) of a single sphere is:

$$
u=u_{\infty}(1-6.55 \nu)
$$


where $u_{0}$ is the velocity of $a$ single sphere in an unbounded fluid and $\nu$ is the volume fraction of the spheres. Drew [1976] combines this with the expression for Stokes drag:

$$
f_{D_{1}}=6 \pi \mu_{f} a u_{0}
$$

where $\mu_{f}$ is fluid viscosity, $f_{0}$ is the force on a single sphere, and $a$ is the syiliere radius, to obtain:

$$
f_{D_{0}}=\frac{6 \pi \mu_{f} a u}{(1-6.55 \nu)}
$$

The drag force per unit volume on the solid (or dispersed) phase is then:

$$
f_{D}=\nu \frac{f_{D_{s}}}{V_{p}}=\nu \frac{6 \pi \mu_{f} a u}{\frac{\pi}{3} \pi a^{3}(1-6.55 \nu)}
$$

or ${ }^{12}$

$$
f_{D}=\frac{\theta}{2} \frac{\mu}{a^{2}} \nu(1+6.55 \nu) v_{r}
$$

where the settling velocity is set equal to the relative velocity $\left(u=v_{r}\right)$ and all other variables are as previously defined.

Tam [1969) theoretically obtains a correction to Stokes drag law that accounts for the volume fraction of the dispersed phase ${ }^{13}$. The modified expression for drag on a single sphere is:

$$
f_{D_{0}}=6 \pi \mu_{f} a\left[\frac{4+3 \nu+3 \sqrt{8 \nu-3 \nu^{2}}}{(2-3 \nu)^{2}}\right] v_{r}
$$

Apazidis [1985] rewrites equation 159 as:

$$
f_{D}=\nu \frac{f_{D_{1}}}{V_{p}}=\frac{9}{2} \frac{\mu}{a^{2}} \mathcal{F}(\nu) v_{r}
$$

for multiple particles and uses it in his suspension model, $\mathcal{F}(\nu)$ in equation 160 is given by:

$$
F(\nu)=\nu \frac{4+3 \nu+3 \sqrt{8 \nu-3 \nu^{2}}}{(2-3 \nu)^{2}}
$$

Note that this expression diverges as $\nu \rightarrow \frac{2}{3}$.

${ }^{12}$ Using a binomial series we can write:

$$
\left.\frac{1}{(1-6.55 \nu)}=1+6.55 \nu+42.0 \nu^{2}+\alpha \nu^{3}\right)
$$

But since Batchelor's original expression (1,e, the left. hand side of the above equation) ia correct only to order' $\nu$, Drew truncates the series after the aecond term.

${ }^{13}$ The aume exprassion is derived by Brinkman [1947) 22 years earlier, though from a different starting point, [Tam, 1960]. 


\subsubsection{Lift}

An expression for lift in the flow of a mixture is obtained by Drew [1976], who generalized Saftman's result for a single particle to:

$$
f_{L i 1}=\frac{3(6.46)}{4 \pi} \frac{\rho_{j}^{\frac{1}{2}} \mu_{j}^{\frac{1}{2}} \nu}{a}\left|D_{j}\right|^{-\frac{1}{3}} \mathrm{D}_{f}(\mathrm{u}-\mathrm{v})
$$

where $\rho_{f}$ is the density of the fluid, $\mu_{f}$ is the viscosity of the fluid, $\nu$ in the volume fraction of the solid (or dispersed) phase, $a$ is the particle size, $v$ is the velocity of the fluid, $u$ is the velocity of the solid phase, and $D_{f}$ is the symmetric part of the fluid velocity gradient, given by:

$$
\mathrm{D}_{j}=\frac{1}{2}\left[\operatorname{grad} \mathbf{v}+(\operatorname{grad} \mathbf{v})^{T}\right]
$$

Decker [1988] comments that it is not clear that this form will reduce to Safimun's result; nor is it clear what is meant by the absolute value or square root operating on Df. Mc'Tigue et al. [1986] clarifies the definition of $\left|D_{f}\right|^{-\frac{1}{2}}$ by writing the above expression as:

$$
f_{L 1}=\frac{3(6.46)}{4 \pi} \frac{\rho_{f}^{\frac{1}{2}} \mu_{j}^{\frac{1}{2}} \nu}{a}\left(\operatorname{tr} D_{f}^{2}\right)^{-\frac{1}{4}} \cdot D_{f}(u-v)
$$

Magnus (or spin) forces are generally neglected in models of two-phase flow on the basis of order of magnitude arguments [Saffman, 1965; Boothroyd, 1967]. These arguments, however, are only strictly valid for the low Reynolds number (and otherwise idealized) situations used by Saffman (1965) and Rubinow and Keller [1961] to derive their analytic results. At least one experimental study [Aoki et al., 1.979] has stated that at higher Reynolds numbers particle migrations can be explained by Magnus forces. It is by no means certain, on the basis of these arguments, that Magnus forces can be neglected in two-phase flows. We generalize Rubinow and Keller's results in the same manner as Drew generalizes Saftrman's to get:

$$
f_{L \lambda}=\frac{3}{4} \nu \rho_{f}\left(\mathbf{W}_{1}-\mathbf{W}_{f}\right)(\mathbf{u}-\mathbf{v})
$$

where:

$$
\mathbf{W}_{\mathrm{a}}=\frac{1}{2}\left[\operatorname{grad} \mathbf{u}-(\operatorname{grad} \mathbf{u})^{T}\right]
$$

and

$$
W_{1}=\frac{1}{2}\left[\operatorname{grad} v-(\operatorname{grad} v)^{T}\right]
$$

An interaction term of this form was included in the mixture balance equations employed by Atkin and Craine [1976a]. The net lift force on the solid phase is simply the surn of these two effects, or:

$$
\mathbf{f}_{L}=\mathbf{f}_{L 1}+\mathbf{f}_{L \text { a }}
$$

Note that this expression is frame-indifferent. 
McTigue et al. [1986] use an expression for lift in their model that also includes a term of the form:

$$
f_{L}=L D_{f} d i v D_{f}
$$

where $L$ is a constant coefficient and $D_{f}$ is the rate of deformation tensor of the fluid, $D_{f}$, defined above. This lift force is apparently a second order effect and is discussed in relation to 'Ho and Leal' lift in Appendix B.

\subsubsection{Virtual Mass}

Having presented an expression for virtual mass force for the simple case of a single sphere, we now need to specify the coefficient, $C_{v m}$, and the relative acceleration, $\mathbf{a}_{v m}$, for more general situations. The virtual mass force $f_{v m}$ is given by (using Drew's [1979] notation):

$$
\mathbf{f}_{u m}=C_{u m} \rho_{f} \mathbf{a}_{u m}
$$

where $a_{v n}$ should be an objective form of the virtual mass acceleration.

The problem of deriving an objective quantity for the relative acceleration is investigated by Drew and Segel [1071a] and El-Kuissy [1975]. Drew et al. [1979] gives a thorough analysis of this problem and proposes an expression they refer to as the most general objective acceleration for two-phase flow ${ }^{14}$ :

$$
\begin{aligned}
\mathbf{a}_{u m} & =\frac{\partial(\mathbf{u}-\mathbf{v})}{\partial t}+[\operatorname{grad}(\mathbf{u}-\mathbf{v})] \mathbf{u} \\
& +[(\lambda-2) \operatorname{grad} \mathbf{u})+(1-\lambda) \operatorname{grad} \mathbf{v})](\mathbf{u}-\mathbf{v}),
\end{aligned}
$$

where $\lambda=\lambda(\nu)$ and $\lambda(\nu \rightarrow 0)=2, \lambda(\nu \rightarrow \infty)=0$. Later, Drew (1983) proposed another frame-indifferent relative acceleration given by:

$$
\mathbf{a}_{u m}=\frac{D^{\prime} \mathbf{u}}{D t}-\frac{D^{\prime} \mathbf{v}}{D t}=\left[\frac{\partial \mathbf{u}}{\partial t}+(\operatorname{grad} \mathbf{u}) \mathbf{v}\right]-\left[\frac{\partial \mathbf{v}}{\partial t}+(\operatorname{grad} \mathbf{v}) \mathbf{u}\right]
$$

A number of investigators perform numerical studies using equation 171 for relative acceleration in their virtual mass terms (Hacox et al, 1980; Kazimi and No, 1986; Lahey et al., 1980; Thorley and Wiggert, 1985] . Lahey et al. (1980) study the effect of adding virtual mass effects to their numerical model. They numerically solve a onedimensional two-fluid model for the special case of adiabatic air/water bubbly flows through nozzles and diffusers. The model includes the interaction effects of drag and virtual mass. The inclusion of a virtual mass term in their model equations did not noticeably change the final numerical reaults, though it did enhance the numerical stability of the system. Hancox et al. [1980] uses equation 171 in their one-dimensional model for gas-liquid flows and finds that it leads to numerical instability resulting in imaginary velocities for some values of $\lambda$. Kazimi and No [1986] include a virtual mass term using equation 171 as the relative acceleration in their two-fluid model. They

\footnotetext{
19The expression given in equation 171 is clearly not the most general expresaion for relative acceleration. 'T he addition of any framenindifferent quantity would always yield a still more 'general' expresaian,
} 
numerically determine values of $C_{v m}$ that yield stability of their numerical solutions, Thorley and Wiggert (1985) use a similar formulatiof 4 gvaluate propagation velocities in a mixture. They conclude that the inclusion of virtual mass effects in their model results in a more accurate and general expression for the acoustic propagation velocity in two-phase media.

Drew and Lahey (1987) also show that the objective form given in equation 171 (with $\lambda=2$ ) reduces to the appropriate expression for the case of a single sphere. ${ }^{16}$

As diacussed enrlier, for a single, non deformable, spherical particle $C_{v m}=\frac{1}{2}$. For two-phase flows, it is reasonable to assume that this coefficient would depend on the void fraction. However, its functional form has not been extensively studied and is not well known; many workers use the value of $\frac{1}{2}$ for lack of any better data. Ziatser [1964] attempts to estimate the effect of concentration on induced mass. With the assumption of uniform concentration Zuber obtains the expression:

$$
C_{\nu m}=\frac{2 \pi}{3} a^{3} \nu \frac{1+2 \nu}{1-\nu}
$$

where $a$ is particle radius and $\nu$ is volume fraction of the dispersed phase. Clearly this expression indicates that virtual mass effects increase with increasing solids concentration. That effect is observed experimentally by Mokeyev [1977] using an electrohydrodynamic analog to simulate two-phase flow in which the fluid is ideal. Mokeyev obtains an empirical function:

$$
\begin{array}{r}
\frac{C_{v m}}{\frac{4}{3} \pi a^{3} \nu}=0.5+2.1 \nu \\
0.10 \leq \nu \leq 0.42
\end{array}
$$

and observes that $C_{v m}$ is constant for $\nu \leq 0.19$. Ishii and Mishima [1984] note that equations 173 and 174 compare quite favorably.

$\mathrm{It}$ is apparent from the expression given in equation 173 that the significance of the virtual mass effect is very dependent upon volume fraction. Clearly, it also increases for larger particles. What is not necessarily evident from this equation is that the relative importance of the virtual mass also depends upon the density of the phases, being most important when the particles are light (less dense). Immich [1980] defines a parameter $\Lambda$ for estimating the contribution of virtual mass as:

$$
\Lambda=\frac{\frac{1}{2} \frac{\rho_{1}}{\rho_{0}}}{1+\frac{1}{2} \frac{e_{1}}{\rho_{0}}}
$$

where $\rho_{d}$ is dispersed phase density and $\rho_{\mathrm{c}}$ is continuous-phase density. $\Lambda$ behaves as a weighting function, varying between 0 and 1. Both Immich [1980] and Shapiro

\footnotetext{
${ }^{15}$ In the same paper, Drew and Lahey (1887) also propose a frame-indifferent expression that incorporates both lift and virtual mass. While the entire expression is frame-indifferent, the individual terms representing lift and virtual mass effects are not. This seems questionable in that each term describes a different physical phenomenon, both of which may or may not be present in a given flow. For instance, in a steady flow where virtual mass effects are not present, one would still expect the expreasion (i.e., for the lift force only) to yield a frame-indifferent quantity for the interactions.
} 
(1986) use this parameter to weight their virtual mass expressions in studying impulsive motions of suspensions. ${ }^{1 \mathrm{~s}}$

\subsubsection{Basset Force}

When a particle is part of a suspension, Zuber [1964) proposes that the Basset force should be increased by a factor of $(1-\alpha)^{-2.5}$. Wallis [1969] suggests that this overestimates the effects of neighboring particles. Anderson and Jackson [1.967) ussume that the Basset force is negligible for a 'large assernbly' of particles. Evaluation of the actual significance of the Basset force in two phase flows awaits further work as no experimental or numerical studies are currently available.

\subsubsection{Buoyancy}

When body forces are present and the components of the mixture have different densities, there will be a buoyant force acting between the phases. While it is important to include the buoyancy term in the force balance on a single particle, in mixture theory the body force terms in the balance of linear momentum equations incorporate this effect. There should not be a buoyancy term in the interaction force vector.

\subsubsection{Diffusion}

Consider the gravity driven sedimentation of a single particulate phase in a liquid. Several investigators study this problem from the standpoint of both averaged fleld equations [Anderson and Jackson, 1967; Drew and Segel, 1971b; Drew, 1976] and mixture theory [Thacker and Lavelle, 1977; Thacker and Lavelle, 1978; Hill et al., 1980] (see Hill et al. [1980] for additional references). Thacker and Lavelle [1978] study the linear stability of the governing equations for sedimentation derived from mixture theory. They find that the solutions of the equations are unconditionally unstable. The addition of viscous, virtual mass, and inertial interaction forces to the momentum equations does not affect the stability of the solutions. Drew and Segel [1971.b] obtain a similar result using averaged equations. Hill et al. [1980] are able to obtain stable solutions of the mixture equations by including diffusive interaction forces in the momentum balances. Clearly one would expect a viable two-component (phase) flow model to, at least qualitatively, describe a relatively simple situation such as sedimentation. We conclude that to obtain well-posed and meaningful problems in mixture theory one must include an interaction term of the form:

$$
f_{d f}=\alpha_{1} \operatorname{grad} \rho_{1}+\alpha_{2} \operatorname{grad} \rho_{2}
$$

for a two component mixture. Here, $\alpha_{1}$ and $\alpha_{2}$ are constants and $\rho_{1}$ and $\rho_{2}$ are the densities of the mixture components. Müller's [1968] work (discussed previously) leads to the same conclusion using a different approach. These densities are related to the

\footnotetext{
${ }^{16}$ Cook and Thomas [1984] study the virtual mass effects for a mixture of a bulk liquid, bubbles, and the liquid associated with each bubble.
} 
densities of the components, taken by themselves, through:

$$
\begin{gathered}
\rho_{1}=\rho_{f}(1-\nu) \\
\rho_{2}=\rho_{s} \nu
\end{gathered}
$$

Assuming the constituents are incompressible, equation 176 may be rewritten as:

$$
\mathbf{f}_{d f}=\left(\alpha_{2}-\alpha_{1}\right) \operatorname{grad} \nu
$$

Note that this is a frame-indifferent quantity [Chadwick, 1976]. This kind of interaction term may also be thought of as accounting for Brownian motion [Drew, 1976] ${ }^{17}$

Leighton and Acrivos [1986, 1987a,b] measure what they refer to as the shearinduced diffusion coefficient in suspensions of spheres. It is not clear precisely how this quantity relates to $\alpha_{1}$ and $\alpha_{2}$, since there appears to be some lift effect due to the fluid shear.

\section{Conclusion}

The flow behavior of fluid-solid mixtures in transport lines has been of interest in chemical processes for many years. In general, empirical relations that predict the flow and pressure drop in such processes have been developed for specific ranges of solids and gas properties as well as for various geometries. A traditional way of modeling the behavior of fluids or suspensions that cannot be modeled by the classical Newtonian fluid model is by non-Newtonian models that are homogeneous. The theory of multiphase mixtures present another avenue of research which can account for the inherent non-homogeneities in the problem.

The theory of interacting continua (or mixture theory) is a means for studying the interaction between several constituents by generalizing the equations and principles for a single continuum. These balance equations express properties common to all materials and motions and hence the differences among the various components that make up the mixture must be augmented in the theory by constitutive relations. Mathematically, the purpose of constitutive relations is to supply connections among kinematic, mechanical, and thermal fields that are compatible with the balance equations and that, in conjunction with them, provide a theory that is solvable for properly posed problems. In mechanics, a constitutive relation is a restriction upon forces, or motions, or both. Postulating constitutive equations in more complex situations, such as multip.'1ase flows, requires a deeper understanding of the nature of the problems. Application. of mixture theory to fluid-particle systems, such as a fluidized bed or fluid-solid transport, requires constitutive relations to determine, for example, the stress of each phase and the interaction forces.

In this work, we have reviewed the existing constitutive relations for these interactive forces. The emphasis has been on the particle-fluid interactions. The general

\footnotetext{
${ }^{17}$ For a discussion of Brownian motion, see Batchelor [1976] and Probstein [1989]. A comparison of Brownian and turbulent diffusion is given in a study by Ounis and Ahmadi [1989].
} 
approach, in multiphase flow studies, has been generalization of the problem of a single spherical particle immersed in an infinite fluid medium by introducing a volume fraction dependence for coefficients such as drag and virtual mass. To mesh and merge the traditional approach with the mixture theory representation of these interaction mechanisms, we have first reviewed the literature on the dynamics of a single particle in a fluid continuum. The work reviewed includes theoretical as well as experimental efforts to formulate appropriate forms for the forces acting on a particle. We then present a review of studies based on continuum theories of mixtures. Various forms for the interactive forces have been given within this context for a variety of problems, such as mixture of ideal gases, mixture of two fluids, flow through porous media, fluidized beds, etc. Finally, we show the attempts, made by various investigators, to generalize the results, both theoretical and experimental, of a single particle to a cloud or an assembly of particles by including a volume fraction (or concentration) dependence in the material properties, such as viscosity, or coefficients, such as the drag coefficient.

A summary of the available results for each interaction force is given below (Also see Table 2):

- Drag acts in the direction of flow and should always be included in any model of two-component flow.

- Diffusion acts in the direction of density gradients. Little is known about the form of the coefficients, but we speculate (on the basis of the stability analyses discussed previously) that a term of this form should be included for all situations.

- 'Slip-Shear' Lift acts perpendicular to the direction of flow. Its importance increases with increasing volume fraction, relative velocity, fluid density, fluid viscosity, and fluid velocity gradients. It cannot be neglected in most situations.

- 'Spin' Lift acts perpendicular to the direction of flow. Though typically a much smaller effect than 'slip-shear' lift, it may become significant at some points in the system (especially for larger particle sizes, because $\mathbf{W}_{\mathbf{s}}-\mathbf{W}_{f}$ becomes larger). Its magnitude increases with increasing volume fraction, fluid density, and relative velocity.

- Virtual Mass effects are present only if there is relative acceleration between the mixture components. The virtual mass force acts in the direction of flow and becomes larger with increasing volume fraction and particle size. Its relative magnitude, in comparison to the other forces accelerating the particles, depends upon the relative densities of the components. It is especially important for large particles that are less dense than the fluid; for example large air bubbles in a liquid medium.

- Basset Force is present only in unsteady (or accelerating) flows. It acts in the direction of flow and increases with increasing particle size, fluid density, and flüiu viscosity. It has been suggested (but not verified) that it also increases with volume fraction. 
The chief conclusion of this review is that for laminar flow of a mixture consisting of an incompressible fluid with entrained solid particles one may write the interaction force of the solid phase on the fluid as:

$$
\begin{aligned}
\mathbf{f}_{I}= & \left(\alpha_{2}-\alpha_{1}\right) \operatorname{grad} \nu+\alpha_{3}(\mathbf{u}-\mathbf{v})+\alpha_{4} \mathbf{D}_{f}(\mathbf{u}-\mathbf{v}) \\
& +\alpha_{5}\left(\mathbf{W}_{6}-\mathbf{W}_{f}\right)(\mathbf{u}-\mathbf{v})+\alpha_{6} \mathbf{a}_{v m}
\end{aligned}
$$

where $\nu$ is the solid volume fraction, $\mathbf{u}$ is solid velocity, $\mathbf{v}$ is fluid velocity, $\rho_{f}$ is the fluid density, $\rho_{0}$ is the solid density, $g$ is the acceleration of gravity (or other body force), $\alpha_{1}$ and $\alpha_{2}$ are unknown coefficients, and:

$$
\begin{gathered}
\mathbf{D}_{f}=\frac{1}{2}\left[\operatorname{grad} \mathbf{v}+(\operatorname{grad} \mathbf{v})^{T}\right] \\
\mathbf{W}_{f}=\frac{1}{2}\left[\operatorname{grad} \mathbf{v}-(\operatorname{grad} \mathbf{v})^{T}\right] \\
\mathbf{W}_{\bullet}=\frac{1}{2}\left[\operatorname{grad} \mathbf{u}-(\operatorname{grad} \mathbf{u})^{T}\right] \\
\alpha_{3}=\frac{9}{2} \frac{\mu_{f}}{a^{2}} \mathcal{F}(\nu) \\
\alpha_{4}=\frac{3(6.46)}{4 \pi} \frac{\rho_{f}^{\frac{1}{2}} \mu_{f}^{\frac{1}{2}} \nu}{a}\left(\operatorname{tr} \mathbf{D}_{f}^{2}\right)^{-\frac{1}{4}} \\
\alpha_{5}=\frac{3}{4} \rho_{f} \nu \\
\alpha_{6}=\frac{2 \pi}{3} a^{3} \nu \frac{1+2 \nu}{1-\nu} \\
\mathcal{F}(\nu)=\nu(1+6.55 \nu)
\end{gathered}
$$

From right to left, the terms on the right-hand side of equation 180 reflect the presence of diffusion (non-uniform solid distribution), drag, 'slip-shear' lift, 'spin' lift and virtual mass. We are neglecting the Basset force for the present because its significance in flows with many particles has not been established; evidence suggests that it is important only for special cases. If the flow is steady, virtual mass effects may also be neglected (i.e. $\alpha_{6}=0$ ). 


\begin{tabular}{||c|c|c|c|c|c|c||}
\hline \multicolumn{7}{|c|}{ Table 2. Effect of Flow Properties on Interactions } \\
\hline \hline Interaction & $\begin{array}{c}\text { Volume } \\
\text { Fraction }\end{array}$ & $\begin{array}{c}\text { Relative } \\
\text { Velocity }\end{array}$ & $\begin{array}{c}\text { Fluid } \\
\text { Viscosity }\end{array}$ & $\begin{array}{c}\text { Fluid } \\
\text { Density }\end{array}$ & $\begin{array}{c}\text { Particle } \\
\text { Size }\end{array}$ & $\begin{array}{c}\text { Velocity } \\
\text { Gradient }\end{array}$ \\
\hline Drag & $\nu(1+6.55 \nu)$ & $\mathbf{u}-\mathbf{v}$ & $\mu_{f}$ & 0 & $\frac{1}{a^{2}}$ & 0 \\
Diffusion & $\operatorname{grad} \nu$ & $?$ & $?$ & $?$ & $?$ & $?$ \\
'Slip-Shear' Lift & $\nu$ & $\mathbf{u}-\mathbf{v}$ & $\mu_{f}^{\frac{1}{2}}$ & $\rho_{f}^{\frac{1}{2}}$ & $\frac{1}{a}$ & {$\left[\mathrm{tr}_{\mathrm{L}} \mathrm{D}_{f}^{2}\right]^{-\frac{1}{2}} \mathrm{D}_{f}$} \\
'Spin' Lift & $\nu$ & $\mathbf{u}-\mathbf{v}$ & 0 & $\rho_{f}$ & 0 & $\mathrm{~W}_{f}-\mathrm{W}_{f}$ \\
Virtual Mass & $\nu \frac{1+2 \nu}{1-\nu}$ & 0 & 0 & 0 & $c^{3}$ & $a_{v m}$ \\
Basset Force & $(1-\nu)^{-\frac{2}{2}}$ & 0 & $\mu_{f}^{\frac{1}{2}}$ & $\rho_{f}^{\frac{1}{2}}$ & $a^{2}$ & 0 \\
\hline
\end{tabular}

There are obvious 'gray' areas in the above information that await further studies for clarification. For instance, there have been no investigations as to what form $\alpha_{1}$ and $\alpha_{2}$ may have. Also, it is not clear if and when the Magnus ('spin' lift) effect is significant. The remaining coefficients have not been extensively studied for general two-phase flows; thus, the forms given above are ad hoc applications of results that are strictly valid under more restricted conditions. Despite the assumptions involved, however, these expressions do provide a qualitative model of how the interaction forces vary with the system parameters. The coefficients can be considered functions of $\nu$ only (because $\nu$ varies with position in the flow) for the purpose of performing numerical studies; the remainder of the expression is then treated as an arbitrary constant to be studied as a parameter in the problem. 


\section{A Frame-Indifference and Constitutive Equations}

\section{A.1 Introduction}

The differences among the materials that make up different bodies are reflected in the theory by constitutive relations. In mechanics, a constitutive relation is a restriction on the forces or the motion of the body or both. This means that a body undergoes a motion when forces act on it, but the motion "caused" depends on the nature of the body. Mathematically, the purpose of the constitutive relations is to supply connections between kinematic; mechanical, and thermal fields that are compatible with the balance equations and that, in conjunction with them, provide a theory that is solvable for properly posed problems. The assumption that the body force is external in a constitutive relation. Indeed, the forces of most interest in continuum mecharics are contact forces, which are determined from the stress tensor field $T$. The mechanical behavior of real materials is very diverse and complex; it is impossible to formulate equations capable of describing the stress in a body under all circumstances. However, just as different figures in geometry are defined as idealizations of natural objects, continuum mechanics seeks to establish particular relations between the stress tensor and the motion of the body for "ideal materials" [Truesdell and Noll, 1965] .

These equations describe the most important features of the behavior of a material in a given situation. In some instances, it may be necessary to represent the same real material by different ideal materials in different circumstances. A classic example is that of the theory of incompressible viscous fluids, which gives an excellent description of the behavior of water flowing through pipes, but is useless for the study of the propagation of sound waves through water. While a constitutive equation is a postulate or a definition from the mathematical standpoint, physical experience remains the first guide, perhaps reinforced by experimental data. Very rarely is it possible to formulate the basic equations of a theory from physical insight only. However, once the theorist has collected the information he wishes to use in defining the ideal materials to be used in his theory, a list of mathematical principles, some perhaps really only guidelines, become essential. in formulating definite constitutive equations [Truesdell and Toupin, $1960]$.

Constitutive relations are required to satisfy some general principles. First, they should hold equally in all inertial coordinate systems at any given time (often referred to as coordinate invariance requirement). This guards against proposing a relation in which a mere change of coordinate description would imply a different response in the material. Many of the so-called "power law" models used in describing non-Newtonian fluids are not invariant. In general, this difficulty can easily be overcome by stating the equations either in tensorial form or by using direct notations not employing coordinates a.t all.

Another principle that is often used as a guide for selecting constitutive parameters is the principle of equipresence. Truesdell and Noll [1965] state this principle as: 
A quantity present as an independent variable in one constitutive equation should be so present in all, unless, of course, its presence contradicts some law of physics or rule of invariance.

Müller [1968] uses this principle in proposing constitutive equations for a mixture of two fluids. However, Rivlin [1972] discusses some cases where this principle seems to be contradictory.

The principle of material frame-indifference (sometimes referred to as objectivity), which requires that the constitutive equations be invariant under changes of frame, is perhaps the most important of all. It is a consequence of a fundamental principle of classical physics that material properties are indifferent, that is, independent of the frame of reference of the observer. A good example of the intuitive concept of this principle is given by Truesdell and Noll [1965] :

A body of known weight, say one pound, when suspended by a given spring is observed to extend it by a given amount, say one inch. The spring and weight, still connected, are then laid upon a horizontal disc, to the center of which the free end of the spring is attached. The disc is then caused to spin at a steady speed such as to extend the spring again by one inch. The spectators are expected to agree that the centripetal force required to hold the weight from flying off is exactly one pound. That is, the response of the spring is unaffected by a rigid motion.

This principle requires that constitutive relations depend only on frame-indifferent forms (or combinations thereof) of the variables pertaining to the given problem.

\section{A.2 Change of Frame}

The fundamental quantities that are measured in kinematics are distance and time intervals. The position of an event la pair $(\mathbf{x}, t)$ consisting of a point $\mathbf{x}$ in space and a time $t$ ] such as velocity, acceleration, or some property can be defined only if a reference frame is specified. Truesdell and Noll [1965] state that "a frame of reference may be described as a possible way of relating physical reality to a three-dimensional Euclidean point space and a real time axis." Two examples of a frame of reference are the fixed stars and the walls of a laboratory. The frame of reference should not be understood as a synonym for coordinate system. In particular, the concept of a change of reference frame should not be confused with a change of coordinate system. A change of coordinate system is only concerned with the spatial variable $x_{;}$a change of frame is concerned with space and time. A change of frame is defined as a one-toone mapping of space-time $(x, t)$ onto itself, which preserves the following properties: (a) the distance between an arbitrary pair of points, (b) the time interval between an arbitrary pair of instants, and (c) the order in which two distinct instants occur.

A change of frame can be expressed as:

$$
\mathbf{x}^{*}=\mathbf{c}(t)+\mathbf{Q}(t) \mathbf{x},
$$




$$
t^{*}=t-t_{0}
$$

where the $x^{*}, t^{*}$ denote position and time in the new frame, and the $x, t$ are position , and time in the old frame. In these equations $\mathbf{c}(t)$ is a vector, $\mathbf{Q}(t)$ an orthogonal tensor [i.e., $\mathbf{Q}(t) \mathbf{Q}^{T}(t)=\mathbf{Q}^{T}(t) \mathbf{Q}(t)=\mathbf{I}$ ], and $t_{0}$ is a real number.

A quantity that remains invariant under all changes of reference frame is called frame-indifferent or objective. In particular, we have the following transformation laws:

1. A scalar remains unchanged under changes of frame.

2. An objective vector $\mathbf{v}$ transforms according to:

$$
\mathbf{v}^{*}=\mathbf{Q}(t) \mathbf{v}
$$

3. A frame indifferent second-order tensor $\mathbf{S}$, regarded as a linear transformation, is one that transforms objective vectors into objective vectors. Thus, an objective tensor transforms according to:

$$
\mathbf{S}^{*}=\mathbf{Q}(t) \mathbf{S Q}^{T}(t)
$$

Functions whose values are scalars, vectors, or tensors are called frame-indifferent or objective if both the dependent and independent variables transform according to the above rules. Thus, if:

$$
\mathrm{T}=\hat{\mathrm{T}}(a, \mathbf{v}, \mathbf{S})
$$

where $a$ is a scalar, $\mathbf{v}$ is a vector, and $\mathbf{S}$ is a second-order tensor, then if $\mathbf{T}$ is to be frame-indifferent, we must have:

$$
\mathrm{T}^{*}=\hat{\mathrm{T}}\left(a^{*}, \mathbf{v}^{*}, \mathbf{S}^{*}\right)=\hat{\mathrm{T}}\left(a, \mathbf{Q v}, \mathbf{Q S Q ^ { T }}\right),
$$

and thus by Equation 192, we have:

$$
\hat{\mathbf{T}}\left(a, \mathbf{Q v}, \mathbf{Q S Q}^{T}\right)=\mathbf{Q} \hat{\mathbf{T}}(a, \mathbf{v}, \mathbf{S}) \mathbf{Q}^{T} .
$$

\section{A.3 Examples}

Having introduced the concept of frame-indifference (objectivity), we now present examples of how one determines frame-indifference of an expression. Though the concepts employed are quite general, we will consider frame-indifferent relative accelerations.

A change of frame is defined by:

$$
\mathbf{x}^{\prime \prime}(\mathbf{X}, t)=\mathbf{c}(t)+\mathbf{Q}(t) \mathbf{x}(\mathbf{X}, t)
$$


as in the previous section. Here $\mathbf{X}$ denotes the current configuration of the body and $\mathbf{X}$ denotes the reference configuration. Taking two material time derivatives of equation 196 we obtain [Truesdell and Noll, 1065] :

$$
\mathbf{v}^{*}(\mathbf{X}, t)=\frac{\partial}{\partial t} \mathbf{X}^{*}(\mathbf{X}, t)=\mathbf{Q}(t) \mathbf{v}(\mathbf{X}, t)+\dot{\mathbf{Q}}(t) \times(\mathbf{X}, t)+\dot{\mathbf{c}}(t)
$$

and:

$$
\begin{aligned}
\mathbf{a}^{*}(\mathbf{X}, t)=\frac{\partial}{\partial t} \mathbf{v}^{*}(\mathbf{X}, t)= & \mathbf{Q}(t) \mathbf{a}(\mathbf{X}, t)+2 \dot{\mathbf{Q}}(t) \mathbf{v}(\mathbf{X}, t) \\
& +\ddot{\mathbf{Q}}(t) \mathbf{X}(\mathbf{X}, t)+\ddot{\mathbf{c}}(t)
\end{aligned}
$$

where the dots denote time derivatives. Note that the velocity can be written in terms of either the reference configuration, $\mathbf{X}$, or the current configuration, $\mathbf{X}$ :

$$
\mathbf{v}(\mathbf{X}, t)=\hat{\mathbf{v}}(\mathbf{x}, t)
$$

so that a (the material time derivative of velocity) can be written as the two equivalent expressions:

$$
\mathbf{a}(\mathbf{X}, t)=\frac{\partial}{\partial t} \mathbf{v}(\mathbf{X}, t)=\frac{\partial \hat{\mathbf{v}}(\mathbf{x}, t)}{\partial \mathbf{x}} \frac{\partial \mathbf{x}}{\partial t}+\frac{\partial \hat{\mathbf{v}}(\mathbf{x}, t)}{\partial t}
$$

Typically in fluid mechanics, equations are written in terms of the velocity referred to the current configuration, i.e. :

$$
\mathbf{v}=\hat{\mathbf{v}}(\mathbf{x}, t)
$$

Given this definition for $\mathbf{v}$, $\mathbf{a}$ in equation 198 is written as the material time derivative $\left(\frac{D}{D t}\right)$ :

$$
\mathbf{a}=\frac{D \mathbf{v}}{D t}=\frac{\partial \mathbf{v}}{\partial \mathbf{x}} \frac{\partial \mathbf{x}}{\partial t}+\frac{\partial \mathbf{v}}{\partial t}
$$

Equation 198 gives the transformation of a under a change of frame. The partial of $v$ with respect to $t$ then transforms according to:

$$
\frac{\partial \mathbf{v}^{*}}{\partial t}=\mathbf{a}^{*}-\mathbf{L}^{*} \mathbf{v}^{*}
$$

where:

$$
\mathrm{L}=\operatorname{grad} \mathbf{v}=\frac{\partial \mathbf{v}}{\partial \mathbf{x}}
$$

and:

$$
\mathrm{L}^{*}=\operatorname{grad}^{*} \mathrm{v}^{*}=\mathrm{QL} \mathrm{Q}^{T}+\dot{\mathrm{Q}} \mathrm{Q}^{T}
$$

\section{A RELATIVE ACCELERATION THAT : IS NOT FRAME-INDIFFEREN'T}

Consider the relative acceleration given by:

$$
\mathbf{a}_{v m}=\frac{D \mathbf{u}}{D t}-\frac{D \mathbf{v}}{D t}
$$


from equation 198 we see that:

$$
\mathbf{u}_{v m}^{*}=Q \mathbf{u}_{v m}+2 \dot{\mathbf{Q}}(\mathbf{u}-v)
$$

and thus the expression in equation 206 is not frame-indifierent by comparison of equation 207 with equation 191 (which is the deflnition of a frame-indifferent vector).

\section{A RELATIVE ACCELERATION THAT IS} FR.AME-INDIFFERENT

Consider the relative acceleration given by:

$$
\mathbf{a}_{u m}=\left[\frac{D \mathbf{u}}{D t}-(\operatorname{grad} \mathbf{u})(\mathbf{u}-\mathbf{v})\right]-\left[\frac{D \mathbf{v}}{D t}-(\operatorname{grad} \mathbf{v})(\mathbf{v}-\mathbf{u})\right]
$$

which we can rewrite as:

$$
\mathbf{a}_{v m}=\left[\frac{D \mathbf{u}}{D t}-\frac{D v}{D t}\right] \cdots(\operatorname{grad}(\mathbf{v}+\mathbf{u})](\mathbf{u}-\mathbf{v}),
$$

We have, from equation 207:

$$
\left[\frac{D \mathbf{u}}{D t}-\frac{D \mathbf{v}}{D t}\right]^{*}=\mathbf{Q}\left[\frac{D \mathbf{u}}{D t}-\frac{D \mathbf{v}}{D t}\right]+2 \dot{\mathbf{Q}}(\mathbf{u}-\mathbf{v})
$$

from equation 197 :

$$
(\mathbf{u}-\mathbf{v})^{*}=\mathbf{Q}(\mathbf{u}-\mathbf{v})
$$

and from equation 205 :

$$
[\operatorname{grad}(\mathbf{v}+\mathbf{u})]^{*}=\mathbf{Q}[\operatorname{grad}(\mathbf{v}+\mathbf{u})\rfloor \mathbf{Q}^{T}+2 \dot{\mathbf{Q}} \mathbf{Q}^{T}
$$

Using equations 210,211 , and 212 in equation 209 yields:

$$
\mathbf{a}_{v m}^{*}=\mathbf{Q} \mathbf{a}_{v m}
$$

which by comparison with equation 191 verifies that the relative acceleration vector given by equation 208 is frame-indifferent.

\section{B Lift Forces}

\section{B.1 Comparison of 'Slip-Shear' and 'Spin' Lift}

Boothroyd [1967] compares the relative magnitudes of the 'slip-shear' lift derived by Saffman $[1965,1968$ ] and the 'spin' lift derived by Rubinow and Keller [1961]. The 'slipushear' lift on a single particle is written as:

$$
f_{L / s}=6.46 \mu_{j}^{\frac{1}{2}} \rho_{f}^{\frac{1}{2}} a^{2}\left|\frac{d v}{d y}\right|^{\frac{1}{2}}(u-v)
$$


and the 'spin' lift is written as:

$$
\mathbf{f}_{L m}=\pi \rho_{j} a^{a} \Omega \times(\mathbf{u}-\mathbf{v})
$$

Note that $\mathbf{f}_{L m}$ acts in the same direction (perpendicular to the slip velocity) as $f_{L o}$. If we nosume that the particle angular velocity is given by:

$$
\Omega=\frac{1}{2} \frac{d v}{d y}
$$

then equation 215 miay be written:

$$
f_{L m}=\pi \rho_{\rho} a^{3} \frac{1}{2} \frac{d v}{d y}(u-v)
$$

Forming the ratio of $f_{L m}$ to $f_{L a}$ for flow in a pipe yields:

$$
\frac{f_{L m}}{f_{L a}}=0.24 \frac{a}{R}\left[\frac{R e_{f} R}{U} \frac{d v}{d y}\right]^{\frac{1}{2}}
$$

where $R$ is the radius of the pipe, $U$ is a reference velocity, and $R e_{f}$ is the pipe Reynolds number based on $R$ and $U$. Boothroyd concludes from this expression that quite often $f_{L a}$ is much larger than $f_{L m}$.

We wish to carry this one step further and assume that the fluid velocity has a parabolic profile:

$$
v=V_{\max }\left[1-\left(\frac{r}{R}\right)^{2}\right]
$$

With this profile, equation 218 becomes:

$$
\frac{f_{L m}}{f_{L_{a}}}=0.344\left(\frac{a}{R}\right) R e_{j}^{\frac{1}{2}}
$$

at the wall of the pipe $(r=R)$. With the choice of $a=0.01 R$ and $R e_{f}=2000$, we obtain:

$$
f_{L m}=0.154 f_{L 。}
$$

We conclude from this that $f_{L m}$ is generally much smaller than $f_{L s}$ as equation 221 represents a relatively high Reynolds number and a reasonable value for $\frac{a}{R}$. Also, the calculation was done for a particle near the wall, where $f_{L m}$ is expected to be greatest (at least for the assumed velocity profile). Though it appears $f_{L m}$ may generally be negligible, it may increase the observed lift force on a particle as much as $15 \%$; more if the particles are larger than $a=0,01 R$ and/or laminar flow exists at higher Reynolds numbers. It is clearly not advisable to neglect the 'spin' lift interaction without careful consideration of the problem at hand.

We now wish to repeat the above calculations for the general expressions for lift presented in this report. We begin with:

$$
\mathbf{f}_{L m}=\frac{3}{4} \nu \rho_{f}\left(\mathbf{W}_{4}-\mathbf{W}_{f}\right)(\mathbf{u}-\mathbf{v})
$$




$$
f_{L \iota}=\frac{3(\theta, 46) \rho_{j}^{\frac{1}{2}} \mu_{j}^{\frac{1}{2}} \nu}{4 \pi}\left(\operatorname{tr} D_{j}^{2}\right)^{-t} D_{f}(u-v)
$$

Now assume:

$$
\begin{gathered}
u=0 \\
v=V_{\max }\left[1-\left(\frac{r}{R}\right)^{2}\right] \mathbf{e}_{x} \\
\operatorname{tr} D_{f}^{2}=2\left(\frac{d v}{d r}\right)^{2}
\end{gathered}
$$

then:

$$
\frac{\left(f_{L m}\right)_{r}}{\left(f_{L 0}\right)_{r}}=\left.0.5783 \frac{\rho_{j}^{\frac{1}{2}} a}{\mu_{j}^{\frac{1}{2}}}\left(\left|\frac{d v}{d r}\right|^{\frac{1}{2}}\right)\right|_{r=R}
$$

where the subscript $r$ indicates the force acting in the radial direction. This expression can be simplifled to:

$$
\frac{\left(f_{L m}\right)_{r}}{\left(f_{L a}\right)_{r}}=0.8170\left(\frac{a}{R}\right) R e^{\frac{1}{2}}
$$

With $a=0.01 R$ and $R e f=2000$ we obtain:

$$
f_{L, m}=0.360 f_{L, ~}
$$

It seems possible that, with this lift model, $\left(f_{L m}\right)_{r}$ will have a signiflcant effect on the particle distributions in some situations.

\section{B.2 'Ho and Leal' Lift}

Ho and Leal [1974] use an asymptotic expansion to calculate the lift on a neutrally buoyant spherical particle in a simple shear or a Poiseuille flow. Their analysis utilizes the concept of an 'undisturbed' flow (that is, the flow field that would exist if the particle were not present) and arrives ut a luteral force on a particle given by:

$$
\mathbf{f}_{L}=\kappa^{2} \operatorname{Re}\left[\beta G_{1}(s)+\beta \gamma G_{2}(s)\right]
$$

This expression is valid for all undisturbed flow fields of the form $\alpha+\beta z+\gamma z^{2}$, Here, $d$ is the distance between walls, $a$ is some characteristic length scale (such as particle radius), and $\kappa=\frac{s}{d}, \alpha, \beta$, and $\gamma$ are constants, $G_{1}(s)$ and $G_{2}(s)$ are functions of the flow geometry numerically calculated and tabulated by the authors, and $g=\frac{x}{d}$ is the dimensionless $z$ coordinate. The Reynolds number, $R e$, is defined by:

$$
R e=\frac{\rho_{f} v_{c}^{*} a}{\mu_{f}}
$$

where $\rho_{f}$ and $\mu_{f}$ are the clensity and viscosity of the fluid respectively and $\nu_{a}^{*}$ is the velocity scale (j.e., mean flow velocity). 
Me'Tigue et al. [1980] cite the above result in using an expression for lift that includes at term of the form:

$$
f_{L}=L D_{f} \text { div } D_{f}
$$

where $L$ is a constant coefflcient and $D_{f}$ is the rate of deformation tensor of the fluid deffined by:

$$
\mathrm{D}_{f}=\frac{1}{2}\left[\operatorname{grad} \mathrm{v}_{f}+\left(\operatorname{grad} \mathrm{v}_{f}\right)^{T^{\prime}}\right]
$$

They have stated that the term given by equation 232 is a generalization of the lift force (equation 230) derived by Ho and Leal [1874], As this is not an obvious conclusion, we wish to evaluate equation 232 for a flow field of the form $\alpha+\beta z+\gamma z^{2}$ and compare the result to Ho and Leal's expression for lift (equation 230). If:

$$
v_{j}=\left(\begin{array}{c}
\alpha+\beta z+\gamma z^{2} \\
0 \\
n
\end{array}\right)
$$

then:

$$
\begin{gathered}
\operatorname{grad} \mathbf{v}_{f}=\left(\begin{array}{ccc}
0 & 0 & \beta+2 \gamma z \\
0 & 0 & 0 \\
0 & 0 & 0
\end{array}\right) \\
\mathrm{D}_{f}=\frac{1}{2}\left(\begin{array}{ccc}
0 & 0 & \beta+2 \gamma z \\
0 & 0 & 0 \\
\beta+2 \gamma z & 0 & 0
\end{array}\right) \\
\operatorname{div} \mathrm{D}_{f}=\frac{1}{2}\left(\begin{array}{c}
2 \gamma \\
0 \\
0
\end{array}\right)=\left(\begin{array}{l}
\gamma \\
0 \\
0
\end{array}\right)
\end{gathered}
$$

Substituting these expressions into equation 232, we obtain:

$$
f_{L} \mathbf{e}_{z}=\left(2 \gamma^{2} z+\gamma \beta\right) \mathbf{e}_{z}
$$

or:

$$
f_{L}=2 \gamma^{2} z+\gamma \beta
$$

Coubider the case of simple shear for which $\gamma=0$. Equation 235 with $\gamma=0$ implies $f_{L}=0$; however, equation 230 with $\gamma=0$ implies:

$$
\mathbf{f}_{L}=\kappa^{2} \operatorname{Re}\left(\beta^{\prime}\right)^{2} G_{1}(s)
$$

Since $G_{1}(s)$ is not generally equal to zero [Ho and Leal, 1974], it seems that Ho and Leal's result, equation 230 , does not generalize to the form of equation 232 . Though the lift torm given by equation 232 does not appear to correspond to Ho and Leal's result, this is not an argument that it should not be included in the interaction terms for two-phase flow. Pasaman [1986] show日 that the momentum interaction, approximated to the second order, does include a term of this form. 


\section{References}

(1) J.E. Adkins. Non-linear diffusion, 1, diffusion and flow of mixture of thudds, Phil. Trans, Royal Soc, Loridon, 255A:607, 1963,

[2] J.E. Adkins. Non-linear diffusion, 2. constitutive oquations for mixture of isotropic fluids. Phil. Trans. Royal Soc. London, 255A:635, 1963.

[3] G. Ahmadi and V.W. Goldochmidt. Motion of particles in a turbulent fluid - the basset history ierm. Irans, ASME, J, Appl. Mech, 38:561-563, 1971.

[4] T.B. Anderson and R. Jackson. Fluid mechanical descriptions of fluidized beds, 1. equations of motion. Ind. Eng. Chern. Fund., 6:527, 1967.

[5] T.B. Anderson and R. Jackson. Fluid mechanical discriptions of fluidized beds, 2 . stability of the state of uniform fluidization. Ind. Eng. Chem. Fund., 7:12, 1968.

(6) H. Aoki, Y. Kurosaki, and H. Anzai, Study on the tubular pinch effect in pipe flow, Bulletin of the JSME, 22:206-212, 1879.

(7) N. Apazidis. On two-dimensional laminar flows of a particulate suspension in the presence of gravity fleld. Int. ‘. Multiphase Flow, 11:675-698, 1985.

[8] R.J. Atkin and R.E. Craine. Continuum theories of mixtures: Applications. J. Inst, Math. Applications, 17:153-207, 1876.

(9) R.J. Atkin and R.E. Craine. Continuum theories of mixtures: Basic theory and historical development. Quart. Jour. Mech, and Appl. Math., 29:290, 1976.

[10] T.R. Auton. The lift force on a spherical body in a rotational flow. J. Fluid Mech., 183:189-218, $198 \%$.

[11] H.M. Barkla and L,J, Auchterlonie. The magnus or robins effect on rotating apheres. J. Fluid Mech., 47:437-447, 1971.

(12) E. Barnea and J. Mizrahi. A generalized approach to the fluid dynamics of particulate systems, part 1. general correlation for fluidization and sedimentation in solid multiparticle systems. Chem. Engng. J., 5:171-189, 1973.

[13] K.D. Barton and R.S. Subramanian. The migration of liquid drops in a vertical temperature gradient. J. Coll. Interface Sci., 133:211-222, 1989.

[14] A. B. Basset. Treatise on Hydrodynamics. Deighton Bell, London, 1888.

[15] G.K. Batchelor. Sedimentation in a dilute dispersion of spheres. J. Fluid Mech., $52: 245-268,1972$.

[16] G.K. Batchelor. Brownian diffusion of particles with hydrodynamic interaction. J. Fluid Mech., 74:1-29, 1976. 
[17] A. Bedford and D.S. Drumhollex. Recent advances: Theories of immiscible and structured mixtures. Int. J. Eng. Sai, 21:863, 1983.

[18] G. Birkhoff, Hydrodynamics, Greenwood Press, 1960,

(19) R.G. Boothroyd. Flowing Gas-Solid Suspensions, Chapman and Hall, London, $196 \%$.

[20] R.M. Bowen. Theory of mixtures, In Continuum Physics, Vol, 3, p. 1, 1976, Ed. A.C. Eringen.

[21] H. Brenner. Hydrodynamic resistance of particles at small Reynolds number. Advances in Chemical Engineering, 6:287, 1966.

[22] F.P. Bretherton. The motion of rigid particles in a shear flow at low Reynolds number. J. Fhuid Mech., 14:284-304, 1.962.

[23] H.C. Brinkman. A calculation of the viscous force exerted by a flowing fluid on a dense swarm of particles, Appl. Sci. Res., A.1:27-34, 1947.

[24] Y.A. Buevich. Motion resistance of a particle suspended in a turbulent medium. Fluid Dynarnics, 1:119, 1960.

[25] P. Chadwick. Continuum Mechanics. Wiley, 1976.

[26] R. Clift, J.R. Grace, and M.E. Weber, Bubbles, drops, and particles. Academic Press, 1978.

[27] B.D. Coleman and W. Noll. The thermomechanics of elastic materials with heat conduction and viscosity. Arch. Rat. Mech. Anal., 13:167-178, 1963.

[28] T.L. Cook and F.H. Harlow. Virtual muss in multiphase flow. Int. J. Multiphase Flow, 10:691-696, 1984.

(29) S. Corssin and J. Lumley. On the equation of motion for a particle in a turbulent fluid. Appl. Sci. Res., A6:114-116, 1956.

[30] R.E. Craine. Oscillations of a plate in a binary mixture of incompressible newtonian fluids. Int. J. Engng. Sci, 9:1177-1192, 1971.

[31] M.J. Crochet and P.M. Naghdi. On congtitutive equations for flow of fluid through an elastic solid. Int. J. Engng. Sci., 4:383--401, 1966.

[32] R. Decker. The application of single particle hydrodynamics in continuum models of multiphase flow. In Mixing and Demixing Processes in Multiphase Flows With Applications to Propulsion Systems Workshop, NASA: Marshall Space Flight Center, Alabama, Feb. 1988.

[33] C.D. Denson, E.B. Christiansen, and D.L. Salt. Particle migration in shear fields. A.I.Ch.E. Journal, 12:589-595, 1966. 
[34] D.A. Drew. Two phase flows: Constitutive equations for lift and brownian motion and some basic flows. Arch, Rat. Mech. Anal., 62:149-163, 1976.

[35] D.A. Drew. Mathematical modeling of two phase flow. Annual Rev. Fluid Mech., 15:261-291, 1983.

[36] D.A. Drew, L. Cheng, and R.T. Lahey. The analysis of virtual mass effects in two-phase flow. Int. J. Multiphase Flow, 5:233-242, 1979.

[37] D.A. Drew and R.T. Lahey. The virtual mass and lift force on a sphere in rotating and straining inviscid flow. Int. J. Multiphase Flow, 13:113-121, 1987.

[38] D.A. Drew and L.A. Segel. Analysis of fluidized beds and foams using averaged equations. Studies in Applied Math., L:233-257, 1971.

[39] D.A. Drew and L.A. Segel. Averaged equations for two-phase flows. Studies in Applied Maith., L:205-231, 1971.

[40] R. Eichorn and S. Small. Experiments on the lift and drag of spheres suspended in a poiseuille flow. J. Fluid Mech., 20:513-527, 1964.

[41] A. Einstein. A new determination of molecular dimensions. In Theory of the Brownian Movement, pages 36-62. Dover Publications, 1956.

[42] M.M. El-Kaissy. The Thermomechanics of Multiphase Systems with Applications to Fluidized Continua. PhD thesis, Stanford University, 1975.

[43] L.J. Forney, A.E. Walker, and W.K. McGregor. Dynamics of particle-shock interactions: Part ii: Effect of the Basset term. Aerosol Sci. Tech., 6:143-152, 1987.

[44] G. Gouesbet, A. Berlemont, and A. Picart. On the tchen's theory of discrete particles dispersion: Can dense discrete particles disperse faster than fluid particles ? Letters in Heat Mass Transfer, 9:407-419, 1982.

[45] G. Gouesbet, A. Berlemont, and A. Picart. Dispersion of discrete particles by continuous turbulent motions. extensive discussion of the tchen's theory using a two-parameter family of lagrangian correlation functions. Phys. Fluids, 27:827$837,1984$.

[46] A.E. Green and J.E. Adkins. A contribution to the theory of non-linear diffusion. Arch. Rat. Mech. Anal., 15:235, 1964.

[47] A.E. Green and P.M. Naghdi. A dynamical theory of interacting continua. Int. J. Engng. Sci., 3:231-241, 1965.

[48] A.E. Green and P.M. Naghdi. A theory of mixtures. Arch. Rat. Mech. Anal., 24:243-263, 1967. 
[49] A.E. Green and P.M. Naghdi. A note on mixtures. Int. J. Engng. Sci., 6:631-635, 1968.

[50] W.T. Hancox, R.L. Ferch, W.S. Liu, and R.E. Nieman. One-dimensional models for transient gas-liquid in ducts. Int. J. Multiphase Flow, 6:25-40, 1980.

[51] J. Happel and H. Brenner. Low Reynolds Number Hydrodynamics. Noordhoff Int. Pub., 1973.

[52] C.D. Hill, A. Bedford, and D.S. Drumheller. An application of mixture theory to particle sedimentation. Trans. ASME, J. Appl. Mech., 47:261-265, 1980.

[53] J.O. Hinze. Turbulence. McGraw-Hill, 1975.

[54] A.T. Hjelmfelt, J.F. Carney, L.F. Mockros, and S. L. Lee. Dynamic response of a restrained sphere in a fluid. J. Eng. Mech. Div. A.S.C.E., 93(EM1):41-56, 1967.

[55] A.T. Hjelmfelt and L.F. Mockros. Stokes flow behavior of an accelerating sphere. J. Eng. Mech. Div. A.S.C.E., 93(EM6):87-102, 1967.

[56] B.P. Ho and L.G. Leal. Inertial migration of rigid spheres in two-dimensional unidirectional flows. J. Fluid Mech., 65:365-400, 1974.

[57] G.M. Homsy, M.M. El-Kaissy, and A. Didwania. Instability waves and the origin of bubbles in fluidized beds, part 2: Comparison with theory. Int. Journal of Multiphase Flow, 6:305-318, 1980.

[58] R.R. Hughes and E.R. Gilliland. The mechanics of drops. Chemical Engineering Progress, 48:497-504, 1952.

[59] H. Inmich. Impulsive motion of a suspension: Effect of antisymmetric stresses and particle rotation. Int. J. Multiphase Flow, 6:44.l-471, 1980.

[60] M. Ishii. Thermo-Fluid Dynamic Theory of Two-Phase Flow. Eyrolles, France, 1975.

[61] M. Ishii and T.C. Chawla. Local drag laws in dispersed two-phase flow. Technical report, ANL-79-105, NUREG/CR-1230, 1979.

[62] M. Ishii and K. Mishima. Two-fluid model and hydrodynamic constitutive relations. Nuclear Engng. and Design, 82:107, 1984.

[63] M. Ishii and N. Zuber. Drag coefficient and relative velocity in bubbly, droplet or particulate flows. A.I.Ch.E. Journal, 25:843-855, 1979.

[64] R. Jackson. The present status of fluid mechanical theories of fluidization. Chem. Eng. Prog. Symposium Series, 66:3-13, 1970.

[65] R. Jackson. Fluid mechanical theory. In Davidson and Harrison, editors, Fluidization. Academic Press, 1971. 
[66] R. Jackson. Hydrodynamic stability of fluid-particle systems. In Davidson, Clift, and Harrison, editors, Fluidization. Academic Press, 1985.

[67] R.C. Jeffrey and J.R.A. Pearson. Particle motion in laminar verticle tube flow. J. Fluid Mech., 22:721-735, 1965.

[68] M.S. Kazimi and H.C. No. On the formulation of the virtual mass term in two fluid models. Nuclear Eng. and Design, 95:163-170, 1986.

[69] L.J.T.M. Kempers. A thermodynamic theory of the Soret effect in a multicomponent liquid. J. Chem. Phys., 90:6541-6548, 1989

[70] A.R. Khan and J.F. Richardson. The resistance to motion of a solid sphere in a fluid. Chem. Eng. Comm., 62:135-150, 1987.

[71] A.R. Khan and J.F. Richardson. Fluid-particle interactions and flow characteristics of fluidized beds and settling suspensions of spherical particles. Chem. Eng. Comm., 78:111-130, 1989.

[72] R. Kowe, J.C.R. Hunt, A. Hunt, B. Couet, and L.J.S. Bradbury. The effects of bubbles on the volume fluxes and the pressure gradients in unsteady and nonuniform flow of liquids. Int. J. Multiphase Flow, 14:587-606, 1988.

[73] R.T. Lahey. Virtual mass effects in two-phase flows - a review. Trans, Am. Nuc. Soc., 42:665-666, 1982.

[74] R.T. Lahey, L.Y. Cheng, D.A. Drew, and J.E. Flaherty. The effect of virtual mass on the numerical stability of accelerating two-phase flows. Int. J. Multiphase Flow, 6:281-294, 1980 .

175] L.D. Landau and E.M. Lifshitz. Fluid Mechanics. Pergamon, 1959.

[76] M.T. Lawler and P.C. Lu. The role of lift in the radial migration of paricles in pipe flows. In Zandi, editor, Advances in Solid-Liquid Flow in Pipes and Its Applications. Pergamon, 1971.

[77] D. Leighton and A. Acrivos. Viscous resuspension. Chemical Engineering Science, 41:1377-1384, 1986.

[78] D. Leighton and A. Acrivos. Measurement of shear-induced self-diffusion in concentrated suspensions of spheres. J. Fluid Mech., 177:109-131, 1987.

[79] D. Leighton and A. Acrivos. The shear-induced migration of particles in concentrated suspensions. J. Fluid Mech., 181:415-439, 1987.

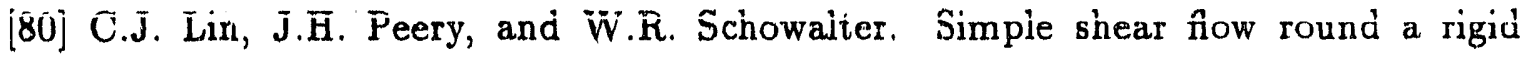
sphere; inertial effects and suspension rheology. J. Fluid Mech., 44:1-17, 1970. 
[81] M. Massoudi and E. Boyle. Continuum theories of granular materials with applications to fluidized beds. Technical report, U.S. Dept. of Energy Report DOE/METC-88/4077, 1987.

[82]' M.R. Maxey and J.J. Riley. Equation of motion for a small rigid particle in nonuniform flow. Phys. Fluids, 26:883-889, 1983.

[83] J.B. McLaughlin. Aerosol particle deposition in numerically simulated channel flow. Phys. Fluids A, 1:1211-1224, 1989.

[84] D.F. McTigue, R.C. Givler, and J.W. Nunziato. Rheological effects of nonuniform particle distributions in dilute suspensions. J. Rheology, 30:1053-1076, 1986.

[85] N. Mills. Incompressible mixtures of newtonian fluids. Int. J. Engng. Sci., 4:97$112,1966$.

[86] Y.G. Mokeyev. Effect of particle concentration of their drag and induced mass. Soviet Research, 6:161-168, 1977.

[87] S.A. Morsi and A.J. Alexander. An investigation of particle trajectories in twophase flow systems. J. Fluid Mech., 55:193-208, 1972.

[88] I. Muller. A thermodynamic theory of mixtures of fluids. Arch. Rat. Mech. Anal., $28: 1-39,1968$.

[89] J.D. Murray. On the mathematics of fluidization, part 1: Fundamental equations and propagation. J. Fluid Mech., 21:465, 1965.

[90] J.W. Nunziato. A multiphase mixture theory for fluid-particle flows. In R.E. Meyer, editor, Theory of Dispersed Multiphase Flow, pages 191-226. Academic Press, 1983.

[91] J.W. Nunziato and D.F. McTigue. Particle segregation in poiseuille flow: A continuum mixture theory. In Proc. IX Int. Congress on Rheology, pages 345352. Mexico, 1984.

[92] J.W. Nunziato, S.L. Passman, R.C. Givler, D.F. McTigue, and J.F. Brady. Continuum theories for suspensions. In Advancements in Aerodynamics, Fluid Mechanics, and Hydraulics, pages 465-472. ASCE Publications, 1986.

[93] F. Odar. Verification of the proposed equation for calculation of the forces on a sphere accelerating in a viscous fluid. Journal of Fluid Mechanics, 25:591-592, 1966.

[94] F. Odar and W.S. Hamilton. Forces on a sphere accelerating in a viscous fluid. Journal of Fluid Mechanics, 18:302-314, 1964.

195] D.R. Oliver. Influence of particle rotation on radial migration in the poiseuille flow of suspensions. Nature, 194:1269-1271, 1962. 
196] H. Ounis and G. Ahmadi. Motions of small rigid spheres in simulated random velocity field. J. Engng. Mech., 115:2107-2121, 1989.

[97] S.L. Passman. Forces on the solid constituent in a multiphase flow. J. of Rheology, 30:1077-1083, 1986.

198] S.L. Passman, J.W. Nunziato, P.B. Bailey, and K.W. Reed. Shearing motion of a fluid.saturated granular material. J. Rheology, 30:167-192, 1986.

[99] S.L. Passman, J.W. Nunziato, and E.K. Walsh. A theory of multiphase mixtures. Technical report, Sandia Report SAND 82-2261, 1983.

[100] W.F. Phillips. Motion of aerosol particles in a temperature gradient. Phys, Fluids, 18:144-147, 1975.

[101] R.F. Probstein. Physicochemical Hydrodynamics. Butterworths, 1989.

[102] R.V. Repetti and E.F. Leonard. Segre-silberberg annulus formation: A possible explanation. Nature, 194:1346-1348, 1964.

[103] R.S. Rivlin. On the principles of equipresence and unification. Q. Appl. Math., $30: 227,1972$.

[104] M.A. Rizk and S.E. Elghobashi. The motion of a spherical particle suspended in a turbulent flow near a plane wall. Phys. Fluids, 28:800-817, 1985.

[105] A. Robinson. On the motion of small particles in a potential field of flow. Comm. Pure Applied Math, 9:69-84, 1956.

[106] D.E. Rosner. Experimental and theoretical research on the deposition dynamics of inorganic compounds from combustion gases. PCH Physico-Chem. Hydrodym., 10:663-674, 1988.

[107] S.I. Rubinow and J.B. Keller. The transverse force on a spinning sphere moving in a viscous fluid. J. Fluid Mech., 11:447-459, 1961.

[108] G. Rudinger. Relaxation in gas-particle flow. In P.P. Wegener, editor, Nonequilibrium Flows, Part 1, pages 119-161. Marcel Dekker, 1969.

[109] P.G. Saffman. The lift on a small sphere in slow shear flow. J. Fluid Mech., $22: 385-400,1965$.

[110] P.G. Saffman. Corrigendum. J. Fluid Mech., 31:624, 1968.

[111] H. Schlichting. Boundary-Layer Theory. McGraw-Hill, 1979.

[112] G. Segre and A. Silberberg. Radial particle displacernents in poiseuille flow of suspensions, Nature, 189:209-210, 1961.

[113] G. Segre and A. Silberberg. Behavior of macroscopic rigid spheres in poiseuille Acw, part 1. J. Flıid Mech, 14:115-135, 1962. 
[114] G. Segre and A. Silberberg. Behavior of macroscopic rigid spheres in poiseuille flow, part 2. J. Fluid Mech., 14:136-157, 1962.

[115] Y.G. Shapiro. Motion of a spherical particle in a stokesian flowfield of a viscous fluid with correction for stokes and saffman forces and also for added mass. Soviet Research, 15:82-94, 1986.

[116] G. Shi. The effect of basset forces on asymptotic particle turbulent diffusivity. In W.W. Bower, editor, Forum on 'lurbulent Flows, pages 35-38. ASME FED Vol. $51,1987$.

[117] J.J.J. Shi, K.R. Rajagopal, and A.S. Wineman. Applications of the theory of interacting continua to the diffusion of a fluid through a.non-linear elastic media. Int. J. Engng. Sci., 19:871-889, 1981.

[118] S.L. Soo. Fluid Dynamics of Multiphase Systems. Blaisdell Publishing, 1967.

[119] S.L. Soo. Equation of motion of a solid particle suspended in a fluid. Phys. Fluids, $18: 263-264,1975$.

[120] S.L. Soo. Net effect of pressure gradient on a sphere. Phys. Fluids, 19:757, 1976.

[121] G.G. Stokes. On the effect of internal friction of fluids on the motion of pendulums. Trans. Cambr. Phil. Soc., 9:8-106, 1851.

[122] J.H. Stuhmiller. The influence of interfacial pressure forces on the character of two-phase flow model equations. Int. J. Multiphase Flow, 3:551-560, 1977.

[123] W.M. Swanson. The magnus effect: A summary of investigations to date. J. Basic Engng., Sept. 1961.

[124] L. Talbot, R.K. Cheng, R.W. Schefer, and D.R. Willis. Thermophoresis of particles in a heated boundary layer. J. Fluid Mech., 101:737-758, 1980.

[125] C.K.W. Tam. The drag on a cloud of spherical particles in low Reynolds number flow. J. Fluid Mech., 38:537-546, 1969.

[126] F.B. Tatum. The basset term as a semiderivative, Applied Scientific Research, $45: 283-285,1988$.

[127] C.M. Tchen. Mean Value and Correlation Problems connected with the Motion of Small Particles suspended in a turbulent fluid. Martinus Nijhoff, The Hague, 1947.

[128] W.C. Thacker and J.W. Lavelle. Two-phase flow analysis of hindered settling. Phys. Fluids, 20:1577-1579, 1977.

[129] W.C. Thacker and J.W. Lavelle. Stability of settling of suspended sediments. Phys. Fluids, 21:291-292, 1978. 
[130] A.R.D. Thorley and D.C. Wiggert. Effect of virtual mass on the basic equations for unsteady one-dimensional heterogeneous flows. Int. J. Multiphase Flow, 11:149-160, 1985.

[131] L.R. Treloar. The Physics of Rubber Elasticity. Oxford, 1975.

[132] C. Truesdell. Sulle basi della thermomeccanica. Rand Lincei, Series 8, 22:33-38 and 158-166, 1957.

[133] C. Truesdell. Mechanical basis of diffusion. J. Chem. Phys, 37:2336, 1962.

[134] C. Truesdell. Rational Thermodynamics. Springer-Verlag, 2nd edition, 1984.

[135] C. Truesdell and W. Noll. The non-linear field theories of mechanics. In Handbuch der Physik (ed. Flugge), Vol. III/3, 1965. Springer-Verlag.

[136] C. Truesdell and R. Toupin. The classical field theories. In Handbuch der Physik (ed. Flugge), Vol. III/1, 1960. Springer-Verlag.

[137] Y. Tsuji, Y. Morikawa, and O. Mizuno. Experimental measurement of the magnus force on a rotating sphere at low Reynolds numbers. Trans. A.S.M.E., J. Fluids Engng., 107:484-488, 1985.

[138] G. B. Wallis. One..Dimensional Two-Phase Flow. McGraw-Hill, New York, 1969.

[139] F.M. White. Fluid Mechanics, McGraw-Hill, 1986.

[140] W.O. Williams. Constitutive equations for flow of an incompressible viscous fluid through a porous media. Quart. Appl. Math., 36:255-267, 1978.

[141] N. Zuber. On the dispersed two-phase flow in the laminar flow regime. Chem. Engr. Sci., 19:897-917, 1964. 
